OPEN ACCESS

Edited by: Gabriella Carini,

Brookhaven National Laboratory (DOE), United States

Reviewed by: Cristoforo Marzocca

Politecnico di Bari, Italy Peter R. Hobson,

Queen Mary University of London,

United Kingdom

*Correspondence:

Klaus Suhling

klaus.suhling@kcl.ac.uk

Specialty section:

This article was submitted to Radiation Detectors and Imaging,

a section of the journal

Frontiers in Physics

Received: 20 December 2019 Accepted: 17 April 2020 Published: 13 May 2020

Citation:

Hirvonen LM and Suhling K (2020)

Fast Timing Techniques in FLIM Applications. Front. Phys. 8:161. doi: 10.3389/fphy.2020.00161

\section{Fast Timing Techniques in FLIM Applications}

\author{
Liisa M. Hirvonen ${ }^{1}$ and Klaus Suhling ${ }^{2 *}$ \\ ${ }^{1}$ Centre for Microscopy, Characterisation and Analysis (CMCA), The University of Western Australia, Perth, WA, Australia, \\ ${ }^{2}$ Department of Physics, King's College London, London, United Kingdom
}

Fluorescence lifetime imaging (FLIM) is increasingly used in many scientific disciplines, including biological and medical research, materials science and chemistry. The fluorescence label is not only used to indicate its location, but also to probe its immediate environment, via its fluorescence lifetime. This allows FLIM to monitor and image the cellular microenvironment including the interaction between proteins in their natural environment. It does so with high specificity and sensitivity in a non-destructive and minimally invasive manner, providing both structural and functional information. TimeCorrelated Single Photon Counting (TCSPC) is a popular, widely used, robust and mature method to perform FLIM measurements. It is a sensitive, accurate and precise method of measuring photon arrival times after an excitation pulse, with the arrival times not affected by photobleaching, excitation or fluorescence intensity fluctuations. It has a very large dynamic range, and only needs a low illumination intensity. Different methods have been developed to advance fast and accurate timing of photon arrival. In this review a brief history of the development of these methods is given, and their merits are discussed in the context of their applications in FLIM.

Keywords: fluorescence lifetime imaging (FLIM), time-correlated single photon counting (TCSPC), photon counting, single photon detection, fast timing

\section{INTRODUCTION}

\subsection{Early Timing of Light Signals}

The quest for precise timing of light signals can be traced back to 1638, when Galileo Galilei (15641642), performed an experiment using two observers with lanterns and manual shutters stationed on two well-separated hilltops. Although he suspected that light did not travel instantaneously, this kind of terrestrial approach was far too slow to observe the speed of light experimentally. Astronomical measurements provided a way forward: In 1675, the Danish astronomer Ole Rømer (1644-1710) observed the eclipses of the innermost moon of Jupiter, Io, at different times of the year and proposed a finite speed of light to account for timing differences of the moon emerging from the shadow of Jupiter, as seen from earth. In addition, in another astronomical approach around 50 years later, in 1728, the British astronomer James Bradley (1693-1762) calculated the speed of light from stellar aberration [1].

First terrestrial measurements of the speed of light were performed by Hippolyte Fizeau (18191896) in 1848-49 by reflecting an intense beam of light from a mirror $8 \mathrm{~km}$ away through a toothed wheel, and in 1850-62 Léon Foucault (1819-1868) improved the experiment by replacing the cog wheel with a rotating mirror, and eventually measured the speed of light within $0.6 \%$ of today's accepted value, and Maxwell's calculations [2]. At King's College London, Charles Wheatstone (1802-1875) also experimented with rotating mirrors to measure the duration of electric sparks [3]. 


\subsection{The Photon}

After the experimental determination of the speed of light, Maxwell's prediction of electromagnetic waves [2] and their experimental detection by Heinrich Hertz in the 1880s, the nature of light came under increased scrutiny. Max Planck's attempt to explain the blackbody spectrum in 1900 ushered in the idea of quantization of energy. Planck considered hypothetical material oscillators that can only emit and absorb electromagnetic radiation in discrete, quantized form and not in continuously varying quantities, especially not in arbitrarily small amounts. Although he did not explicitly propose that light is quantized, he proposed that the energy $\epsilon$ of these oscillators is proportional to their oscillation frequency $v$

$$
\epsilon=h v
$$

where $h$ is now known as Planck's constant. In 1905, Albert Einstein [4] introduced the smallest unit of energy of electromagnetic radiation, Lichtquant (quantum of light), and explained the photoelectric effect using this concept. The term photon was conceived by the chemist Lewis in 1926 [5], and the existence of light quanta as well as the term photon gained wider acceptance as quantum theory was developed in the 1920s.

In particle physics, the photon is an integer spin particle, a boson, with spin 1. A photon is a "quantized field," it always travels at the speed of light and has a momentum, but no mass, and no charge [6]. It does not decay, once it is emitted, its frequency stays fixed ${ }^{1}$.

\subsection{Experimental Detection of Photons and Photon Arrival Timing}

Experimental detection of single photons requires that the signal from a single photon is converted into an electronic signal. The photoelectric effect, first observed by Heinrich Hertz in 1887 [7], provides the means to do this. While the electronic signal of one photoelectron is too small to be detected, the secondary emission principle, discovered by Austin and Starke [8], allows electron multiplication and hence gain to be introduced. Thus, the photoelectron signal, created by a single photon, can be amplified and measured.

The capability to detect single photons means that it is also possible to time their arrival, similar to timing the arrival of particles. Indeed, the origin of time-correlated single photon counting (TCSPC) lies in particle physics. In 1929, Walther

Abbreviations: ADC, Analog-to-Digital Converter; APD, Avalanche Photodiode; CCD, Charge-Coupled Device; CMOS, Complementary Metal Oxide Semiconductor; CFD, Constant Fraction Discriminator; FAD, Flavin Adenine Dinucleotide; FLIM, Fluorescence Lifetime Imaging; FRET, Förster Resonance Energy Transfer; FWHM, Full Width at Half Maximum; IRF, Instrument Response Function; LIDAR, Light Detection and Ranging; MCP, Microchannel Plate; MPPC, Multi-Pixel Photon Counters; NADH, Reduced Nicotinamide Adenine Dinucleotide; NADP, Nicotinamide Adenine Dinucleotide Phosphate; PMT, Photomultiplier Tube; SPAD, Single-Photon Avalanche Diode; TAC, Timeto-Amplitude Converter; TCSPC, Time-Correlated Single Photon Counting; TDC, Time-to-Digital Converter; TIRF, Total Internal Reflection Fluorescence; TTS, Transit Time Spread.

${ }^{1}$ The Nature milestone series "Photons" details background and context of the development of the understanding of light and its basic unit, the photon. http:// www.nature.com/milestones/milephotons/index.html
Bothe and Werner Kohlhörster measured the coincidence of penetrating charged particles in cosmic rays [9] leading to the first practical electronic coincidence circuit [10] which became a precursor of the AND logic circuits of electronic computers. By the addition of a delay, this coincidence method evolved to measure delayed coincidence, providing the means for timeresolved measurements and the invention of the "time circuit," nowadays known as the Time-to-Amplitude Converter (TAC) [11]. It became a popular method to measure short radioactive decay times, with time resolution improved to 80 ps in the $1950 \mathrm{~s}$ [12]. Scintillation decay times, excited by gamma rays, were also reported in the 1950s, using a delayed coincidence method [13].

In 1961, Lowell Bollinger and George Thomas generalized the scintillation measurements to include any type of radiation [14], and flashlamps with optical pulse widths of around 2 ns became available in the 1960s, enabling TCSPC: This is essentially a delayed coincidence method, whereby the arrival time of a single photon is measured relative to an excitation pulse, and this can be done with a few picosecond precision [15]. The accumulation of the arrival time of many single photons then represents the intensity decay of the sample, as long as no photons are lost due to pile-up, and the linearity between intensity and collected photons holds. See Figure $\mathbf{1}$ for a timeline of the development of single photon counting and timing technology.

\subsection{Fast Timing in Fluorescence}

In fluorescence measurements, the sample is excited with a short pulse of light and the decay of fluorescence is measured. This approach can be traced back to the phosphoroscope developed in 1857 by Alexandre-Edmond Becquerel (1820-1891), based on a similar principle to Foucault's speed of light measurement experiments. Becquerel used two rotating disks with a series of holes, and the sample in between, to measure photoluminescence lifetimes. In his apparatus, the sample (e.g., a phosphorescent crystal) was excited by light coming in through one hole, and viewed by the phosphorescent light coming out of the other hole. By varying the speed of rotation, Becquerel was able to measure the short microsecond time interval during which the phosphorescent light is emitted.

In modern time-correlated single photon counting experiments, the photons emitted by the sample are timed one by one and used to build a histogram of the fluorescence decay over time, see Figure 2A. In the simplest case, the fluorescence decay is a monoexponential function

$$
I(t)=I_{0} e^{-\frac{t}{\tau}}
$$

where $I_{0}$ represents the fluorescence intensity at $t=0$ [16]. The fluorescence decay time $\tau$ is the average time the fluorophore remains in the excited state and is defined by

$$
\tau=\frac{1}{k_{r}+k_{n r}}
$$

where $k_{r}$ is the radiative rate constant which is largely determined by the extinction coefficient, the fluorescence spectrum and the refractive index of the fluorophore's environment [17]. The nonradiative rate constant $k_{n r}$ typically depends on the environment, 


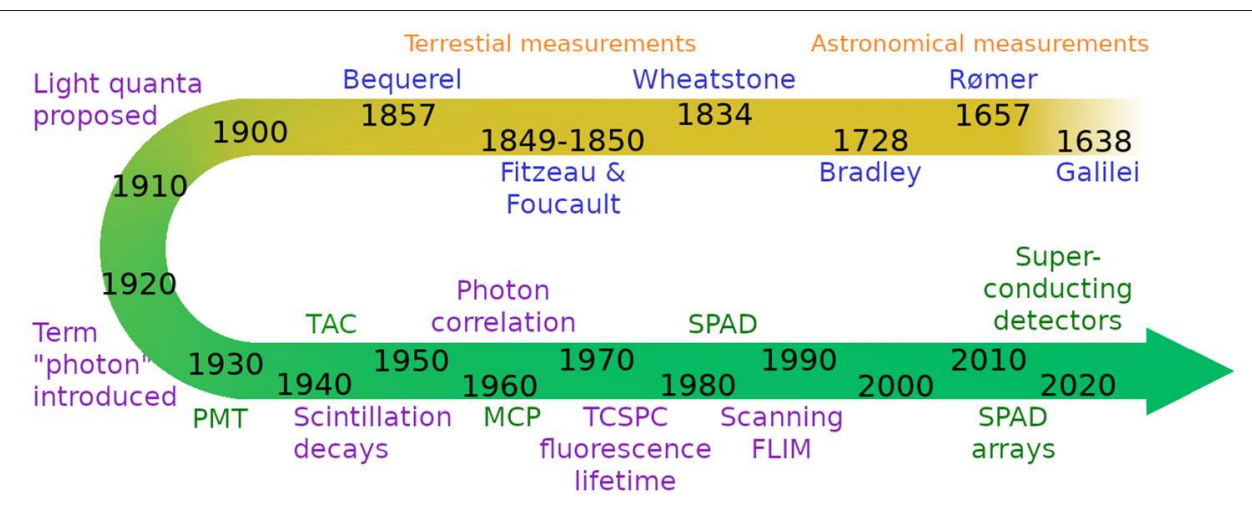

FIGURE 1 | Brief schematic timeline of single photon counting and timing technology from Galilei's work on the speed of light to today's photon detectors and timing electronics. Scientists are indicated in blue, ideas and concepts in purple, and technology developments in green.

and can change with interaction, $\mathrm{pH}$, viscosity, and other parameters [16].

Fluorescence decay measurements in fluorescence spectroscopy or fluorescence microscopy can therefore be used to gain information about the immediate environment of the fluorophore, as well as to probe the proximity of other fluorophores via Förster Resonance Energy Transfer (FRET) [1820]. Moreover, the fluorescence decay is typically independent of the fluorophore concentration at concentrations low enough to avoid interaction or aggregation.

The first reports that use TCSPC in the measurement of fluorescence decays appear in the early 1970s [21-24], and TCSPC was soon widely used for time-resolved spectroscopy, and in particular the measurement of fluorescence lifetimes in solution [25].

Photon counting techniques are used in many different fields of science and technology, from DNA sequencing [26] to quantum cryptography and interplanetary communications [27], and photon timing applications include light detection and ranging (LIDAR) [28, 29], photon correlation techniques [30], and optical tomography [31]. Single photon detection techniques and applications have been reviewed recently by Buller and Collins [32], Hadfield [33] and Eisaman et al. [34]. Incidentally, the same technology is not only used for photon arrival timing, but also particle arrival timing for particles such as neutrons, where timing can indicate neutron energy $[35,36]$, and electrons and ions, e.g., in ion velocity mapping [37-39], so there is a growing need for this technology, [40] with applications beyond timing the arrival of photons.

\subsection{Fluorescence Lifetime Imaging (FLIM)}

The development of laser scanning confocal microscopes [41] enabled TCSPC-based fluorescence lifetime imaging (FLIM), as shown in Figure 2B. This method was developed in the late 1980s [42] and early 1990s [43-45] before being more widely applied in the late 1990s [46]. Besides scanning with a point-detector to form an image of the sample, microchannel plate (MCP)-based detectors and more recently single photon avalanche diode (SPAD) arrays can be used for wide-field TCSPC FLIM [47]. The development of both single point and widefield single photon detection methods has continued over the decades, and while the old technologies continue to be used and improved, there have also been new developments that show great promise, including SPAD arrays and detectors based on superconducting detector technology. See Figure $\mathbf{1}$ for a timeline of single photon technology.

The specific requirements for the detectors and the timing electronics depend on the field of application. This review gives an overview of the technology behind the different approaches to fast photon timing and recent developments with a focus on fluorescence decay measurements and particularly FLIM.

\subsection{Principles Behind Photon Timing}

Photon timing means that the arrival time of a photon at the detector is measured with respect to some reference, typically the excitation laser pulse. A trigger from the laser and the signal from the detector are fed into the timing circuit and their difference is calculated. The photons are then usually assigned a "time bin," i.e., a slot whose width usually depends on the detector precision, the required measurement precision, and the total length of the decay to be measured, see Figure 3. The circuit is adjusted such that the entire decay fits into the available time window, typically 5 times the length of the fluorescence lifetime, so that it can decay into the background noise. The measurement is repeated for many photons, until the arrival time histogram-representing the fluorescence decay-contains a statistically significant number of photons for fluorescence decay analysis, and to extract the fluorescence lifetime [48-51].

\subsubsection{Instrument Response}

A photon timing detector is usually characterized by the full width at half maximum (FWHM) of its instrument response function (IRF), which is measured using a very short pulse of light. In an ideal case, the measured IRF would be a Dirac delta function in a single time bin, but due to experimental uncertainties it is always wider. The width of the instrumental response $\Delta t_{I R F}^{2}$ is given by the sum of the squares of the individual time spread contributions [52]

$$
\Delta t_{\text {IRF }}^{2}=\Delta t_{\text {optical }}^{2}+\Delta t_{t t s}^{2}+\Delta t_{j i t t e r}^{2}
$$



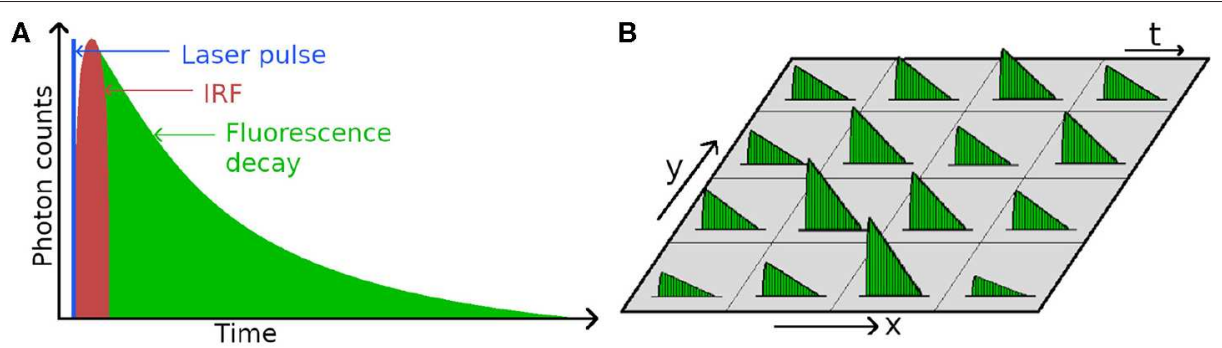

FIGURE 2 | (A) Schematic diagram of a fluorescence decay, (B) Basic principle of Fluorescence Lifetime Imaging (FLIM), where a fluorescence decay is measured for every pixel of the image.
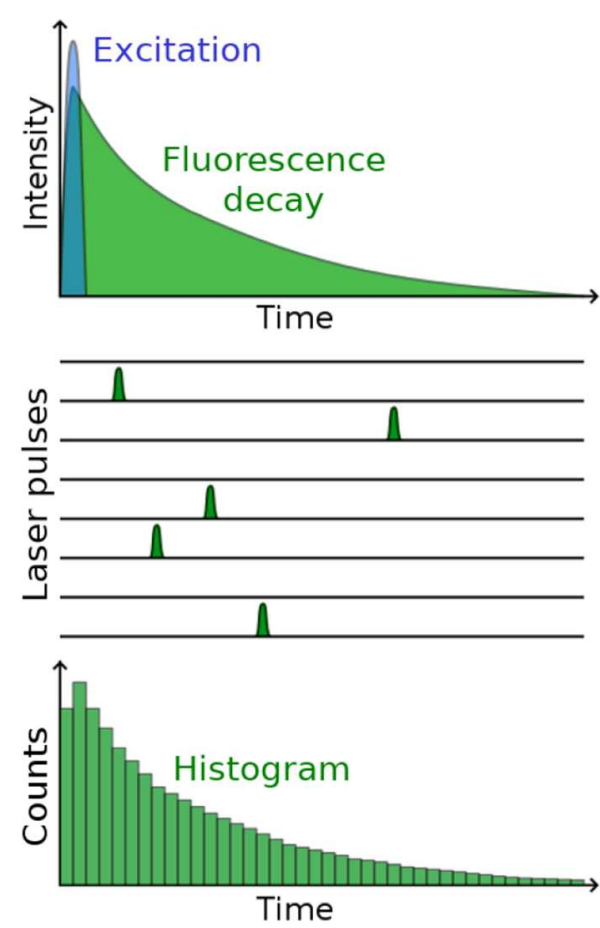

FIGURE 3 | Principle behind photon arrival timing for TCSPC. After repeatedly exciting the sample and timing the arrival of many fluorescence photons (schematically indicated in the middle), a histogram is built up that represents the fluorescence decay.

where $\Delta t_{\text {optical }}$ is the optical pulse width of the exciting light, $\Delta t_{t t s}$ is the transit time spread of the detector, and $\Delta t_{j i t t e r}$ is the jitter of the detection and timing electronics. Optical pulse widths can be as short as femtoseconds, e.g., for two-photon excitation, and the jitter of the electronics can be around 1 ps for the fastest timing systems.

\subsubsection{The Poisson Statistics of Photon Counting}

One key advantage of photon counting is the well-defined theoretical framework of Poisson statistics, which govern the photon counting process. The photon events are independent, i.e., in ensemble measurements the detection of one photon event does not change the probability of detecting another one. (For single photon work, this idea is modified, and in Hanbury Brown and Twiss-type experiments, this is exploited to gain information about coincident events.) The variance is the mean of the distribution, and the experimental uncertainty in the form of the standard deviation is given by the square root of the number of counts. This also means that the more photons are collected, the smaller the experimental uncertainty, and in practice the dynamic range is only limited by the detector and electronics non-linearities. Poisson statistics also predict how often multiple photon events are detected as a function of the mean count rate. Importantly, the probability that more than one photon occurs after an excitation event is never zero, and there are excitation cycles where this happens. If only one photon can be detected after an excitation event, then the first photon arriving is timed, but subsequent photons are lost. This is known as pile-up which distorts the fluorescence decay toward shorter times [15]. In practice, in the older spectroscopy literature it is recommended to keep the stop-to-start ratio (ratio of fluorescence photon count rate to repetition rate of the excitation laser) to around $1 \%$, for fluorescence decays with typically 10,000 counts in the peak [15].

Again, it is worth emphasizing that pile-up always occurs, the probability to detect several photons after one excitation pulse is never zero-the question is whether the effect emerges from the Poisson noise (square root of the number of counts). For FLIM, with typically fewer counts in the fluorescence decays in each pixel than in spectroscopy measurements, the 1\% stop-to-start ratio can be somewhat relaxed, and a $10 \%$ stop-to-start ratio only introduces a $2.5 \%$ fluorescence lifetime error [49]. If all photons that are emitted after an excitation event are detected, then this is not a problem [53].

\section{SINGLE PHOTON DETECTORS}

The detection of a single photon requires that (1) the photon is converted into an electronic signal, and (2) that this signal is larger than the noise, so it can easily be measured-in practice, it needs to be amplified. Different detectors have different characteristics regarding transit time spread, detection efficiency, noise, ease-of-use and durability. Detectors used in TCSPC/FLIM applications are discussed in this section, see Table 1 for a summary. 
TABLE 1 | Summary of single photon detectors that are, or could be, used in FLIM.

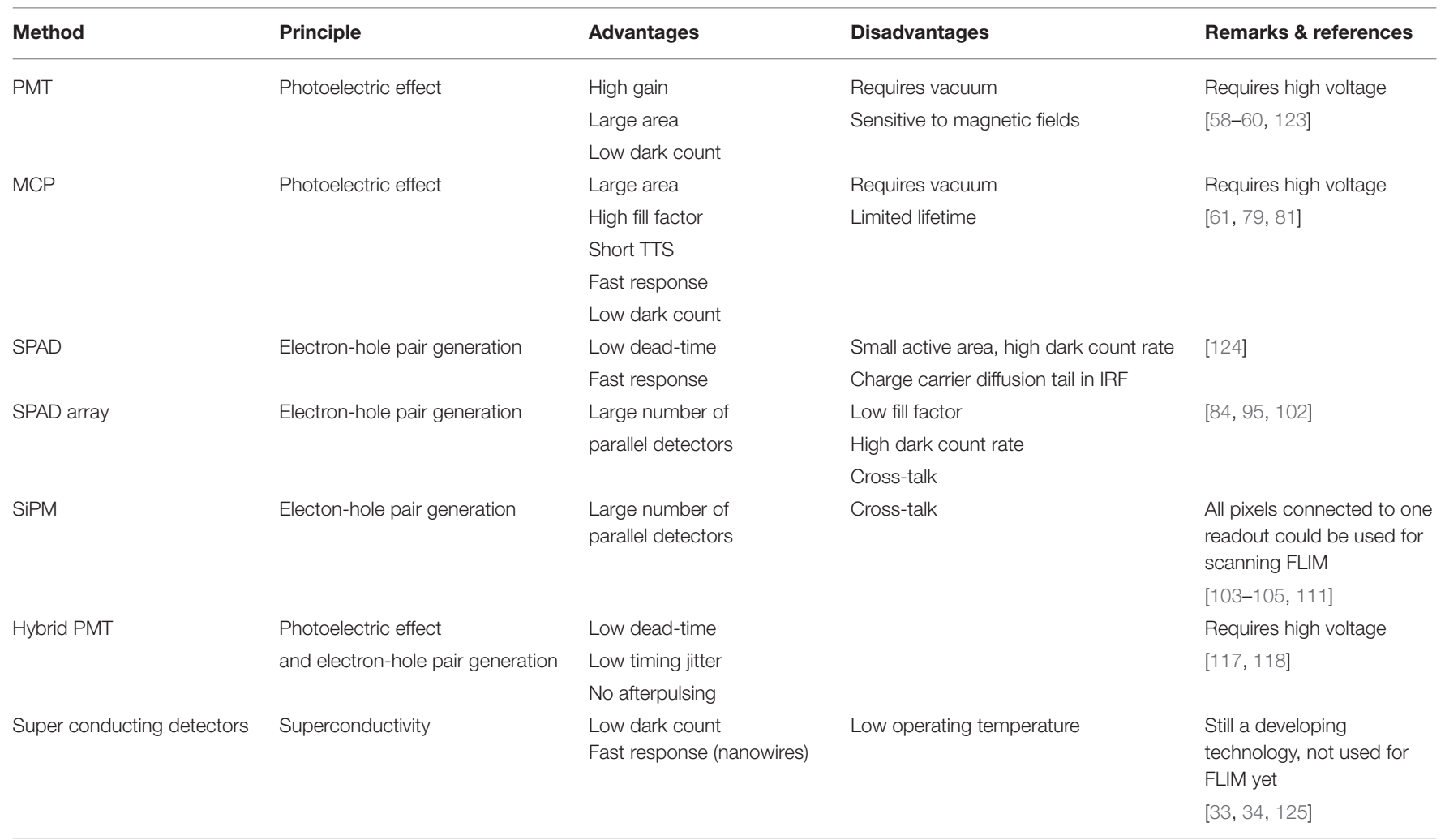

\subsection{Photomultiplier Tube (PMT)}

The first working PMTs were produced in the former Soviet Union in the mid-1930s [54-56] in the race to develop electronic television, and were soon shown to be single photon sensitive [57]. A PMT is a vacuum device that consists of a photocathode, up to a dozen discrete dynodes, each at a higher potential than the previous one, and an anode, see Figure 4 [58-61]. A photon hitting the photocathode can liberate a photoelectron, which in turn releases secondary electrons when it hits the first dynode, attracted to move toward it by the positive potential. The electric signal is amplified in the same way at each dynode, and the overall gain of a PMT can reach values of $10^{6}$ to $10^{8}$ which yields an easily measurable current at the anode, or a pulse. If two photons are detected in quick succession, the output pulses can merge and increase the pulse height. This effect has been exploited to design a so-called pulse pile-up inspector that rejects pulses over a certain pulse height and thus reject multiphoton events which can bias a fluorescence decay $[21,62]$.

The reproducibility of measurements is affected by the electron transit time, anode pulse rise time, and electron transit time spread. The transit time spread determines the full width half maximum of the IRF. For example, according to its data sheet $^{2}$, the Hamamatsu R14755U PMT's single photon pulse has a rise time of $400 \mathrm{ps}$, but a transit time spread of only $200 \mathrm{ps}$. Short rise times are advantageous for precise timing. Incidentally, the IRF is much smaller than the width of the single-photon output

$\overline{{ }^{2} \text { https://www.hamamatsu.com/eu/en/product/type/R14755U-100/index.html }}$ pulse of the PMT, which is quoted as $680 \mathrm{ps}$. The spectral response characteristics are determined on the long wavelength side by the photocathode material and on the short wavelength side by the window material. PMTs require a vacuum and a high voltage for their operation, and are sensitive to strong magnetic fields.

Photon detection efficiency of PMTs depends strongly on the photocathode. The detection efficiency of conventional bialkali and multialkali cathodes reaches $20-25 \%$ between 400 and $500 \mathrm{~nm}$, but falls off quickly toward longer wavelengths. GaAsP cathodes can reach up to $50 \%$ detection efficiency and work up to $700 \mathrm{~nm}$. However, dark current also depends on the cathode material and can become a problem especially with materials that have increased sensitivity toward infrared wavelengths. Typical dead-times of PMTs are in the order of $10 \mathrm{~ns}$, which is generally lower than the dead-time of the electronics used for photon timing and therefore usually not a concern for photon timing applications. They also usually have a large detector area compared to diodes $-50 \mathrm{~cm}$ diameter for the Super-Kamiokande PMTs [63] - which makes them easy to align in confocal or multiphoton excitation fluorescence microscopes. Multianode PMTs have been produced, with 16 anodes, and have been employed in spectral FLIM $[64,65]$.

\subsection{Microchannel Plate (MCP)}

The concept of a microchannel plate (MCP) with a continuous dynode was conceived in the 1930s [66] (coincidentally around the same time as PMTs), although the first working devices were not produced until the early 1960s [67-69]. Like PMTs, 


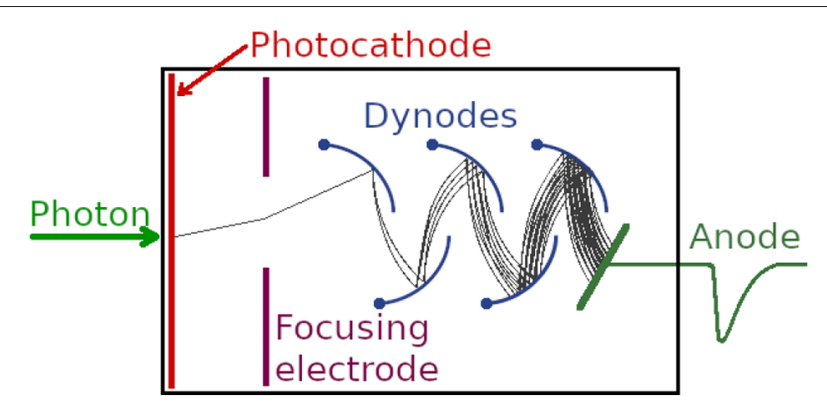

FIGURE 4 | Schematic of a photomultiplier tube. A photon impinging on the photocathode can liberate a photoelectron which is attracted to the first dynode, where further electrons are created by secondary emission. After around 10 dynodes, the gain can be well over $10^{6}$, producing an output pulse that can be timed by timing electronics [58-61].

the operating principle of MCPs is also based on the signal from the emission of a photoelectron from a photocathode and its amplification via secondary emission, but an MCP has continuous dynodes rather than discrete dynodes, as shown in Figure 5. This shortens the transit time, and hence also the transit time spread, which is a critical parameter for photon timing applications. MCPs have a regular array of tiny tubes (microchannels) where electrons are accelerated from one side of the plate to the other through a high voltage (typically a few $\mathrm{kV}$ ). The microchannels are usually straight and round with $\sim 10 \mu \mathrm{m}$ in diameter with a center-to-center distance of $\sim 15 \mu \mathrm{m}[70,71]$. They are arranged at an angle toward the normal (bias angle), so that ion feedback is minimized. Most MCP detectors consist of two (chevron configuration) or three (z-stack configuration) MCPs either pressed together or with a small gap between them, and the bias angles of the plates are rotated $180^{\circ}$ with respect to each other to prevent ion feedback. One MCP can have gain up to 10,000 , but a 3-MCP intensifier can provide gain $>10^{7}$.

MCP detectors do not in principle have "dead-time" when the detector is not capable of detecting new events. However they can be affected by gain depletion where electrons are not delivered fast enough to an area where many events occur, leading to reduced electron gain and consequently reduced output signal amplitude. This can become an issue especially with camerabased read-out which can detect up to 100 s of events per frame [47], but this is unlikely to become an issue if the MCP is used for timing photons one by one.

Inside MCPs the electron transit time is short, and consequently, the transit time spread is also short, 10 s of ps. Indeed, MCPs are used as fast detectors for timing in TCSPC, and IRFs of $<20$ ps have been reported $[48,51]$. The output electron cloud can then be detected by several methods, depending on the application.

If no position read-out is required, the total current can be converted to a voltage via a resistor and read out as a pulse, as schematically indicated in Figure 5. For wide-field TCSPC, a position-sensitive read-out is necessary, and the electrons can be detected with either a position-sensitive anode, or converted to photons with a phosphor screen and detected with a camera

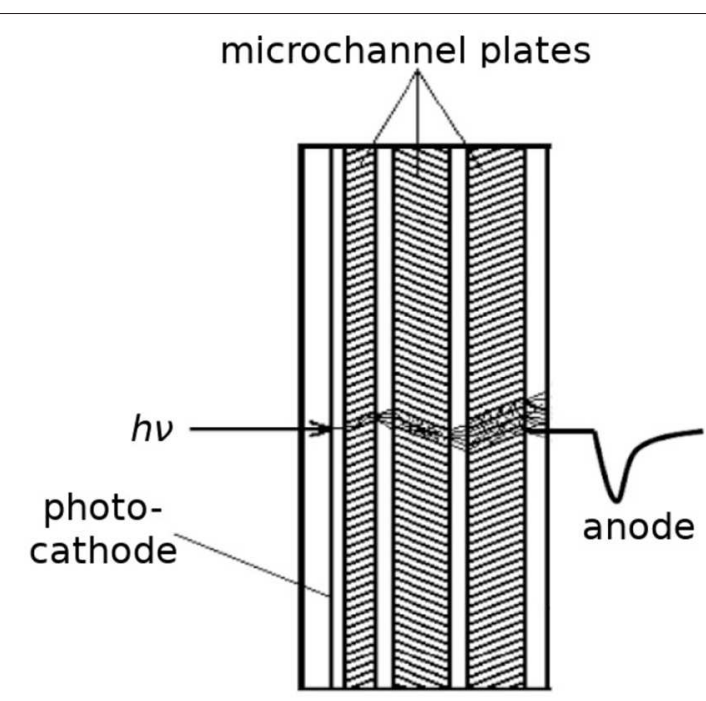

FIGURE 5 | Schematic of a microchannel plate. A photon impinging on the photocathode can liberate a photoelectron, which is attracted into the first microchannel plate where secondary emission inside the microchannels produces more electrons. They can be converted into a timing pulse at the anode.

[47]. Large area MCPs with dimensions of $20 \times 20 \mathrm{~cm}^{2}$ have been reported [72].

Although MCPs are a mature technology, they are still widely used and their development continues. For example, unlike PMTs, MCPs can provide spatial resolution, which is advantageous in low light level imaging. Photon counting imaging, where the amplified photon events were imaged with a camera and the image assembled photon by photon, was employed by astronomers due to the exquisite sensitivity this method offered at the time [73]. Indeed, the Hubble space telescope's faint object camera was based on photon counting imaging [74]. The $\mathrm{x}$-ray multimirror satellite's optical monitor [75], the ultra-violet/optical telescope (UVOT) on the Swift gamma-ray observatory [76] and the Galaxy Evolution Explorer (GALEX) satellite [77] all employ MCP-based photon counting image intensifiers. Developments in the field are ongoing [78, 79], and recent improvements using atomic layer deposition include robust substrates that able to withstand high processing temperatures, very low background rates, high stable gains, and low outgassing $[80,81]$.

\subsection{Avalanche Photodiodes (APD/SPAD)}

In contrast to PMTs and MCPs that are vacuum devices operated at $\mathrm{kV}$ voltages, avalanche photodiodes (APDs) are semiconductor solid-state devices based on a $\mathrm{p}-\mathrm{n}$ junction, for which no vacuum and $\mathrm{kV}$ voltages are required. A diode is an electronic component that allows current to flow through it in one direction (when biased in forward mode), but not the other (reverse mode), as schematically shown in Figure 6A. A photodiode has a depletion region which is largely free of mobile charge carriers, but when a photon is absorbed in it, an electron-hole pair is created, see Figure 6B. Applying an electric field (reverse bias) will allow the 
holes to go to the anode, and the electrons to the cathode-thus a flow of current is created by absorbing photons in the depletion region of a photodiode when biased in reverse mode.

By applying a high reverse bias voltage (typically $100-200 \mathrm{~V}$ in silicon), APDs show an internal current gain effect (around 100) due to impact ionization (avalanche effect). Gain can be improved by different doping and beveling techniques. A key feature of the electron-hole pair generation is that unlike in the photoelectric effect, this is a process for which no vacuum is required.

SPADs are APDs that are operated with a reverse voltage above a typical APD's breakdown voltage. This is also called Geiger mode, and it can achieve gains of $10^{5}-10^{6}$. This mode is particularly useful for single-photon detection. Once a photon has been absorbed and an electron-hole pair created, an electron avalanche is initiated which reduces the reverse bias below the breakdown voltage and eventually stops the avalanche. If the SPAD is designed for passive quenching, a large resistor in series to the photodiode quenches the avalanche, whereas in active quenching, an electronic circuit is triggered by the avalanche current to stop it. Single photons thus produce a clear output signal that can be counted and timed. This output signal is typically independent of the number of photons producing electron-hole pairs in the depletion region, so two photons detected in quick succession will not increase the pulse height, and pile-up inspection based on pulse height [21, 62] is not feasible.

SPADs and APDs have a much smaller active area than photomultipliers, typically between 10 and $100 \mu \mathrm{m}$ in diameter. They can have larger quantum efficiency than photocathodebased photoelectronic vacuum detectors, up to about $80 \%$. SPADs have a low dead-time and consequently high count rate, with transit time spread of 10 s of ps comparable to MCPs $[82,83]$. However, they do have a charge carrier diffusion tail (a type of afterpulsing) in the IRF. Another characteristic feature of SPADs is their dark noise, typically tens of counts/s depending on the operating voltage and temperature [84] and is a key difference compared to photoelectronic vacuum devices for which 0.02 events $\mathrm{cm}^{-2} \mathrm{~s}^{-1}$ have been quoted [71]. For typical nanosecond fluorescence lifetime measurements in $50 \mathrm{~ns}$ time windows, this is not a key issue, but the dark noise consideration gains in importance as the detection window and fluorescence lifetime to be measured increase, and the count rate drops. It is also an important point to consider when enlarging the size of the light sensitive area in SPADs, as the dark noise increases with the area [84].

\subsection{Single Photon Avalanche Diode (SPAD) Arrays}

SPADs can be arranged into arrays for spectroscopy or imaging, and can be manufactured by complementary metal oxide semiconductor (CMOS) technologies [85]. One-dimensional arrays [86-91] can have a good fill-factor, since the light-sensitive regions can be adjacent, with the timing electronics placed away from the illuminated area. Two-dimensional SPAD arrays can be used as a camera for direct wide-field imaging, where each
SPAD is a pixel that can independently perform photon arrival time detection via TCSPC [92-94]. One of the features of such an approach is that it yields enormous overall count rates of $\mathrm{GHz}$ [95]. In addition to TCSPC operation, they can be used in gated mode [96]. Two-dimensional arrays built for fast photon timing have a fill factor well below $100 \%$ (e.g., from $\sim 1 \%[97,98]$ to $\sim 50 \%$ [99]) because the majority of the area in each pixel is occupied by electronic circuits to perform the timing. Quantum efficiencies of around $60 \%$ have been reported [100], which compares favorably to photocathode-based devices. Large arrays of SPADs have been demonstrated to be suitable for TCSPC $[95,101]$, and the development of these detectors is currently developing at a fast pace [102].

\subsection{Silicon Photomultipliers (SiPM)}

Silicon photomultipliers, also known as solid state photomultipliers, or multi-pixel photon counters (MPPCs) are arrays of hundreds to tens of thousands of integrated SPADs, each of which can detect photons individually and independently [103-105]. However, unlike in SPAD arrays, they are all connected to a common readout. Upon detection of a photon in a SPAD, an electrical signal is generated by the SPAD. This can be added to the signal from other SPADs that have detected a photon, to generate a pulse whose amplitude is proportional to the number of photons detected by the SiPM-this is the analog operation of the SiPM, which yields information about the photon flux. Alternatively, the number of SPADs that detected a photon can be counted separately, so that the number SPADs producing a signal is proportional to the number of incident photons, or the photon flux.

Interestingly, SiPMs have a photon number resolving capability: one, two, three or more detected photons can easily be resolved [103]. A pulse height distribution of the SiPM output shows characteristic peaks corresponding to the number of photons detected, in addition to picosecond arrival timing. The latter feature has been used in employing a SiPM with an active area of $1 \mathrm{~mm}^{2}$ for TCSPC [106].

One feature of SiPMs and SPAD arrays is optical cross-talk. The detection of a photon by the semiconductor can create an infrared photon, which in turn can be detected by a neighboring SPAD [107-109]. However, while the effect exists in principle, it can be minimized with an appropriate SPAD design, and cross-talk as low as $1 \%$ has been reported [109].

SPAD arrays and SiPMs can be manufactured by CMOS technologies, but are usually produced using an ad hoc technology [85]. This is a photolithographic fabrication process using field effect transistors for logic functions in various integrated circuits, but also for analog circuits in image sensors; for example, the $160 \times 128$ SPAD array in Veerappan et al. [92] has been quoted to have 60 million transistors. While the latter SPAD array was manufactured using $130 \mathrm{~nm}$ CMOS technology, the latest SPAD arrays have been produced in $40 \mathrm{~nm}$ CMOS technology [110], allowing smaller features on the SPAD array. The pixel pitch, for example, $18.4 \mu \mathrm{m}$ in $\mathrm{x}$ and $9.2 \mu \mathrm{m}$ in $\mathrm{y}$ is the smallest one reported for SPAD arrays at the time of writing, much smaller than, for example, the $50 \mu \mathrm{m}$ pitch used in Veerappan et al. [92]. Since the light-sensitive area 

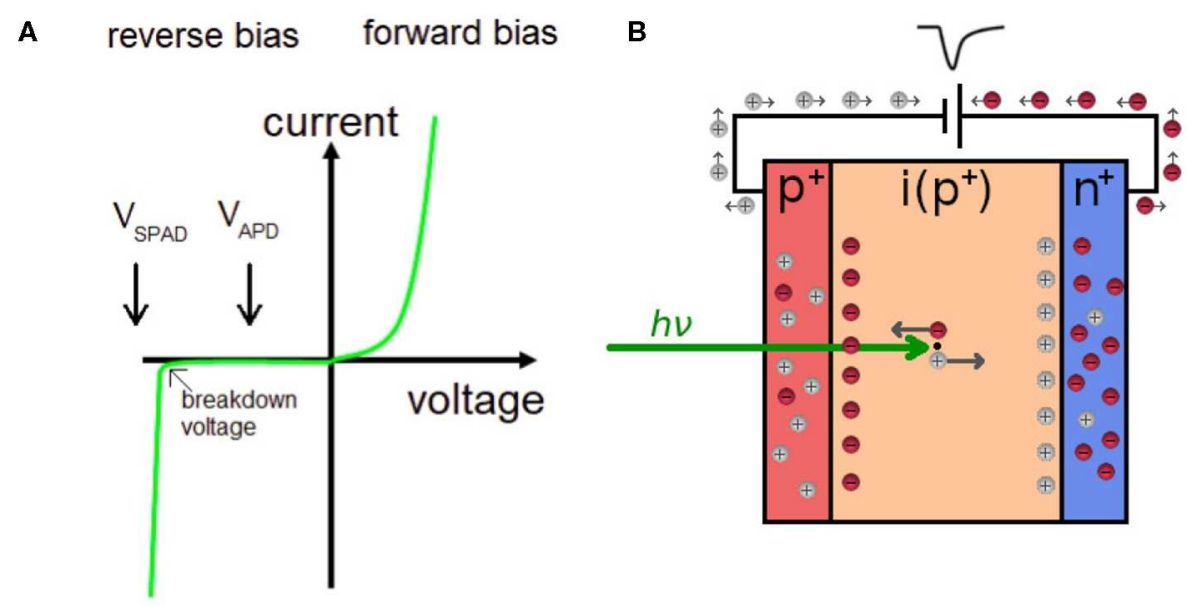

FIGURE 6 | (A) A schematic current-voltage characteristic for a diode. A SPAD is biased above the breakdown voltage of the diode, whereas an APD is biased below the breakdown voltage. (B) A schematic of a photodiode in reverse mode. A photon absorbed in the depletion region (i) creates an electron-hole pair, which through impact ionization produces a pulse for timing.

in a pixel has to share the available space with the timing electronics, the fill-factor (i.e., the ratio of light-sensitive area to total pixel area) is less than $100 \%$, if both are implemented in a two-dimensional layer. This is currently the case, but threedimensional integration, where the light-sensitive part and the electronics are implemented in separate layers which are then put together to form the SPAD array, or SiPM, is an area of active and rapid development, and 100\% fill-factor devices will likely be produced in the near future. This would be a very welcome development for many application areas, including biophotonics $[102,111]$. Three-dimensional integration will also enable placement of FPGA-like (field programmable gate array) structures beneath the sensor, for detailed analysis of the photon flux, e.g., mean fluorescence lifetime determination without reading out the arrival time of every photon [112-114].

\subsection{Hybrid Detectors}

Hybrid photodetectors combine the PMT vacuum tube with photodiode semiconductor technology: a photoelectron liberated from the photocathode is accelerated through a high voltage (up to $10 \mathrm{kV}$ ) directly onto an avalanche diode, biased below the breakdown voltage (see Figure 6A) where it generates a large number of electron-hole pairs, one per each $3.7 \mathrm{eV}$ [115]. Thus, well over 1,000 electron-hole pairs are created by a single photon, which are further amplified in the diode, see Figure 7. One advantage of this technology is that the single gain step produces a narrow pulse height distribution $[115,116]$, which can be used to detect photon numbers. From the timing point of view, this single gain step results in low transit time spread and therefore low timing jitter [117].

Hybrid detectors feature lower dead-times ( $<1 \mathrm{~ns}$ ) compared to either PMTs or SPADs (tens of ns). Also, the IRF of such detectors is, unlike that of a SPAD, PMT or MCP, free of afterpulsing or charge carrier diffusion tails, which reduces counting background and results in a considerably increased

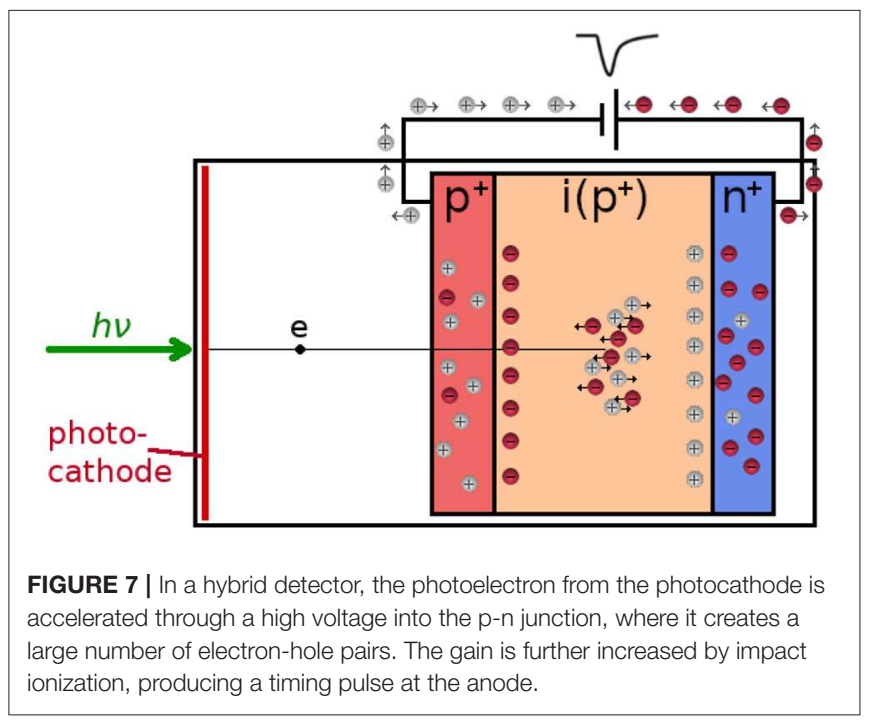

lifetime accuracy for a given number of detected photons $[117,118]$.

Hybrid detectors are now the most commonly used detectors in point-scanning TCSPC FLIM applications. This is due to their high quantum efficiency for GaAsP photocathodes ( 50\%), their large area (compared to SPADs) and fast time resolution (20 ps) [51]. Sixteen channel version of hybrid detectors are available. The principle behind hybrid detectors has also been used to create pixelated detectors for imaging (i.e., electron-bombarded CCDs $[115,119,120]$ and CMOS cameras $[121,122])$ but these devices have not been modified for fast timing applications.

\subsection{Superconducting Detectors}

Superconducting single photon sensors are a relatively new technology. Superconducting nanowire single photon detectors, developed in 2001 [126], have potential for low jitter, short dead-time and high count rates [125]. In combination with 


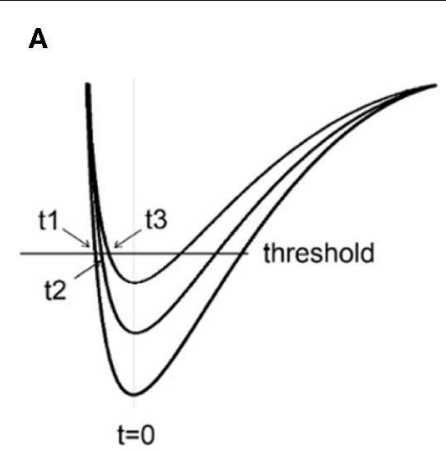

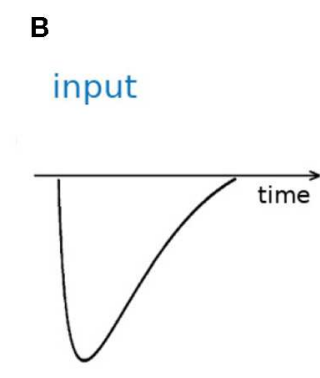

original pulse

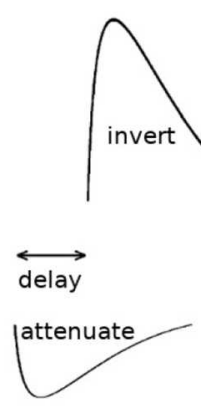

split into two

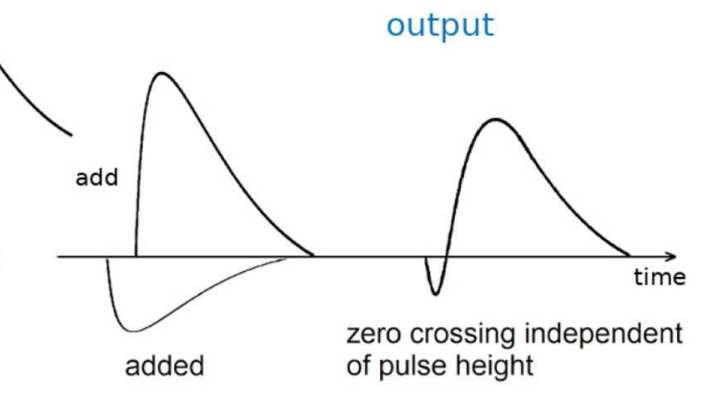

FIGURE 8 | (A) Schematic of leading edge discrimination. The fixed threshold times the pulses (all arriving at the same time $t=0$ ) at different times $t$, $t 2$, and $t 3$, depending on the pulse height. (B) Schematic of constant fraction discrimination. The original input pulse is split into two, one is attenuated, and one is delayed and inverted. The zero-crossing when the two pulses are added is independent of the pulse height.

TCSPC, an IRF of 2.7 ps has been reported, currently the shortest TCSPC IRF on record [127]. Transition edge sensors were first demonstrated in 1995 [128] and have 100 ns jitter and long deadtime making them unsuitable for fast timing applications [129]. The same is true for superconducting tunnel junction detectors, the single photon events have microsecond rise times, depending on the details of the superconducting material. They do have an intrinsic wavelength resolution, though, which is a very attractive feature. The biggest drawback of superconducting sensors is that they have to be operated at liquid helium temperature. Although they are an exciting development in the field of single photon detection, they currently find applications mostly in quantum optics, astronomy and long distance communications [129]. Consequently their development is aimed more toward the infrared wavelengths rather than visible light currently used in FLIM, to the best of our knowledge have not been used for infrared FLIM and are therefore out of scope for this review. For more information, see recent reviews [33, 34, 125, 129].

\section{PHOTON TIMING TECHNOLOGY}

\subsection{Leading Edge and Constant Fraction Discrimination}

After the electronic pulse created by a photon leaves the detector, it may pass through a pre-amplifier before it encounters some kind of discriminator. The discriminator sets the threshold for the pulse to be accepted, and shapes it for detection by the timing circuit. Leading edge discrimination sets a fixed threshold for the incoming pulse, and crossing this threshold provides the timing reference. If the pulses are of a constant amplitude, this is a feasible approach, for example for excitation laser trigger pulses. However, if the pulse height varies, then leading edge discrimination introduces an experimental uncertainty, time walk, which degrades the precision of the timing, as indicated in Figure 8A.

This time walk can be minimized by using a constant fraction discriminator (CFD). A CFD splits the input pulse in two, attenuates one of them, and delays and inverts the other, before they are added again, as shown in Figure 8B. The zero-crossing is independent of the pulse height. Therefore, for pulses for varying pulse height, as is typical for single photon detectors, a CFD minimizes the dependence of the photon arrival timing on the pulse height. The delay is chosen according to the rise time of the leading edge of the pulses to be timed.

\subsection{Time-to-Amplitude Converter (TAC)/ Analog-to Digital Converter (ADC)}

As the earliest photon timing technology, the TAC was derived from the delayed coincidence circuit [11]. It measures the time between a laser pulse and the arrival of a photon, and converts this time difference into a measurable voltage amplitude. A TAC typically starts charging a capacitor upon receipt of a "start" pulse, and stops charging it when a "stop" pulse arrives, such that the capacitor charge is proportional to the time elapsed between "start" and "stop." In practice, to avoid resetting the electronics after every laser pulse, TACs are usually operated in reversed start-stop mode where the arrival of the photon starts the TAC and the arrival of the next laser pulse stops it [130].

To record statistics of photon arrival times (e.g., fluorescence decays), the TAC output pulse, the height of which is proportional to the charge on the capacitor, is processed by an analog-to-digital converter (ADC). The ADC resolves the TAC voltage into a digital equivalent of the photon detection time, which is then used to address a memory location for the arrival time bin and increment it by one count. In early TCSPC systems the ADC was the bottleneck both in terms of speed and channel uniformity. This has been resolved by the development of electronics; the electronics required for TAC/ADC systems are relatively complicated, as the TAC requires a highly linear voltage ramp for precise operation, and the $\mathrm{ADC}$ has to be able to resolve the voltage into thousands of time channels with equal width $[48,49]$. The integral non-linearity of a TAC refers to the actual length of the time window compared to what it should be according to the settings. The differential non-linearity refers to the variation of the counts from time channel to time channel, and this is typically around $1 \%$.

Nowadays TAC/ADC systems can achieve very fast timing in the order of a few ps. In this case the photon timing capabilities 
are usually limited by the timing jitter in the detector (i.e., transit time spread), and, with commonly used ps pulsed diode lasers, the optical excitation pulse width.

\subsection{Time-to-Digital Converter (TDC)}

TDCs are relatively new compared to TAC/ADC technology. As a fully digital system, a TDC counts clock cycles between a start and a stop pulse $[131,132]$. The time resolution of a TDC is determined by the clock speed, typically from a few tens to hundreds of ps at best (e.g., a $1 \mathrm{GHz}$ clock has time resolution of $1 \mathrm{~ns}$ ). In principle, TDC time resolution can be improved by using time interpolation circuits to measure clock cycle fractions, but in this case many advantages, such as simple circuit design and short dead-time are lost.

Although timing resolution of TDCs can be worse than those of TACs, they have some advantages over the TAC/ADC technology. TDCs can have low dead-times in the 1 ns regime, and are favorable in applications where low dead-times are a priority. TDCs can in principle measure any time span, unlike a TAC which has a limited voltage range, and are more robust against environmental changes (e.g., temperature) [53].

TDCs are much easier to upscale than TAC/ADC technology. For this reason TDCs have found widespread use in SPAD arrays, and the development of this technology is expected to continue. Recently a 32-channel TCSPC system has been developed employing the hybrid integration of a custom 32 SPAD array with 32-channel active quench and time to analog converter array $[87,133,134]$. Although the fill-factor and SPAD performance are compromised by having such a large number of detectors and timing electronics on a single substrate, this detector has a TDC in each pixel with 55 ps resolution, allowing independent TCSPC in each pixel of a $32 \times 32$ pixel array simultaneously.

TAC/ADC systems, based on charging a capacitor with a constant current source [135], were and are mainly used in fluorescence spectroscopy systems, as they are a mature technology with the best timing precision and good linearity. However, they have a dead-time that does not make it feasible to time the arrival of two photons in one excitation cycle. TDC systems are based on clock cycles, and typically have a lower time resolution and higher differential and integral non-linearities than TACs, but they have a very short deadtime which allows timing of multiple photon arrivals after one excitation pulse [53]. They are also compact, and TDCs are typically the choice of timing circuit used on TCSPC-based SPAD arrays (although TACs have also been implemented [101]). For implementation in CMOS, on a SPAD array, a stable power supply, the power consumption and the heat generated by its operation, the temperature stability as well as non-uniformity across all pixels are all important considerations [135].

\subsection{Streak Cameras}

Streak cameras differ significantly from the other photon timing methods introduced in this review and are less common, but they are the fastest detectors available and have some uses in FLIM applications where extremely fast timing resolution is needed. Although they can photon count, they do not need
TACs and ADCs or TDCs to perform the photon arrival timing, instead they convert photon arrival time into spatial position on a phosphor screen. They can be considered a more integrated or complete imaging system compared to PMTs, MCPs or SPADs which produce a pulse to be timed by appropriate separate electronics. Similar to image intensifiers, photon detection capability of a streak camera is based on electron amplification inside an MCP, but deflector plates and a voltage sweep circuit are placed between a photocathode and the MCP such that the photoelectrons liberated from the photocathode experience a deflecting voltage before hitting the MCP. The voltage experienced by the electron depends on the photon arrival time at the photocathode, therefore photons arriving at different times end up in different locations on the phosphor screen which is imaged by a camera, see Figure 9. This detection mode is often combined with spectral dispersion in orthogonal direction, such that the image has time distribution in one axis and spectral distribution in the other.

An obvious disadvantage is that one spatial dimension is taken up by the time axis, and the other possibly by spectral detection, so the camera is capable of measuring only one point at a time. It is possible to operate a streak camera in a line scanning mode, where the one spatial dimension in the camera images the time and the other one of the sample spatial directions, and a line is scanned across the sample for a twodimensional image [136, 137]. Alternatively, a streak camera can be combined with a point-scanning confocal or two-photon microscope, where the time and spectral coordinates are obtained from the streak camera and the image is formed by usual pointscanning methods [138-140].

The operation of a streak camera is not necessarily based on single photon detection, although they can be operated in single photon mode [140, 141]. The voltage sweep can be adjusted for the required time resolution and dynamic range-timing resolution in the sub-ps regime is possible with this method. Wider use of streak cameras is limited by their cost, also the time

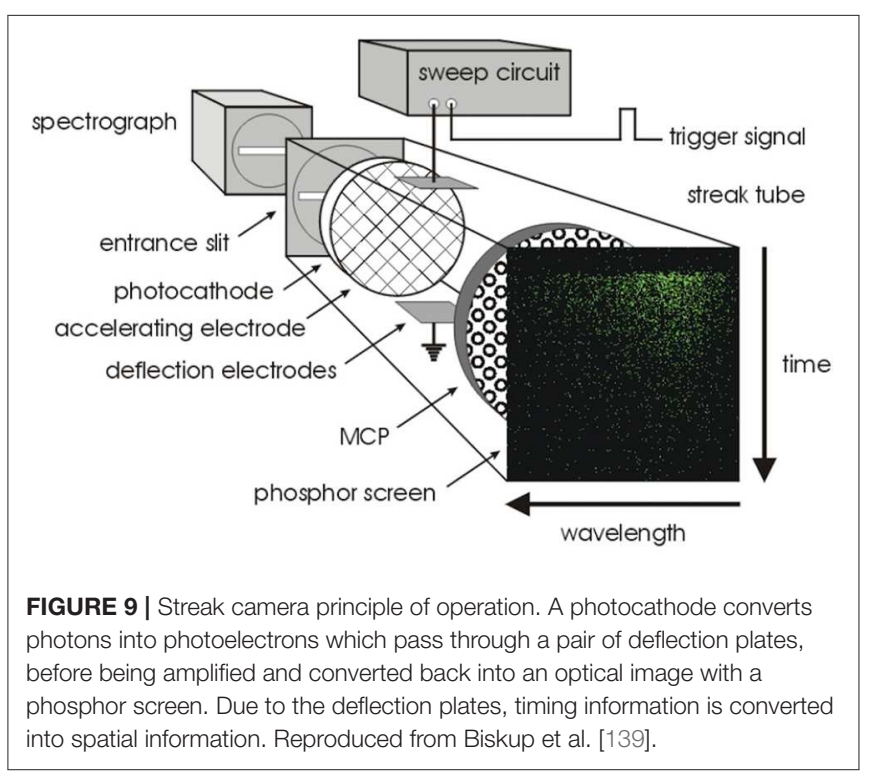


required for scanning an image and data processing to obtain a FLIM image can be lengthy.

\section{FLUORESCENCE LIFETIME IMAGING}

In FLIM microscopy, the timing of photons is used to build a histogram of photon arrival times-which is the fluorescence decay-in each pixel of an image, as schematically illustrated in Figure 2A. The decay is fitted with an exponential decay function (Equation 2) that yields the fluorescence lifetime, which is typically nanoseconds, and often depends on the microenvironment of the molecule, or the proximity of other fluorescent molecules. Therefore FLIM has found many applications, especially in the biological sciences, for monitoring the microenvironment and/or protein interaction [142-145].

When choosing a detector for FLIM, there is no simple answer for the "best" choice-this depends on the microscope and the sample. Fluorescence lifetimes are typically in the order of a few nanoseconds and in some applications IRFs of several hundred ps may be acceptable, but in order to measure complex multiexponential decays, or detect subtle changes in living specimens, fast timing of photon arrival is essential [51].

Apart from timing of individual photons, the time it takes to acquire a whole image is also very important in FLIM. For biological imaging and especially live cell applications, photon efficiency and light dose are critically important. Usually a compromise has to be found between timing accuracy, data acquisition speed and illumination light dose. FLIM methods can be generally divided into two categories: in scanning FLIM a laser beam is scanned over the image pixel by pixel and the image is recorded with a point detector (Figure 10A), whereas wide-field FLIM collects decays for the pixels in parallel fashion (Figure 10B) [47, 146].

\subsection{Scanning FLIM}

While the earliest fast fluorescence timing experiments were point measurements, usually measuring solutions in a cuvette, the development of laser scanning confocal microscopes [41] enabled TCSPC-based FLIM in the 1990s, and opened up new applications for FLIM in biological research. In scanning FLIM a laser beam is scanned over the image pixel by pixel, and the image is recorded with a point detector such as a PMT,
SPAD or a hybrid detector. For proper analysis of the decay, a histogram is required for each pixel of the image, with good photon statistics in each pixel. The excitation repetition rate is fundamentally limited by the fluorescence lifetime to be measured so that the sample has time to decay to the ground state before a new excitation pulse, and to avoid photon pileup, the detection rate is usually limited to $1 \%$ of the excitation repetition rate at the brightest pixel. This can make image acquisition slow, typically a few minutes per image. Scanning FLIM is widely used, and it provides the benefits of confocal or multiphoton excitation microscopy, i.e., optical sectioning, reduced background blur and reduced photobleaching outside the focal point. Combination with spectral detection or polarization is also relatively straight-forward.

One limiting factor in acquisition speed is the dead-time of the detector and the timing electronics; this is the time required to reset the electronics, during which any arriving photon will be lost. PMTs can have dead-times of several tens of ns, while SPADs and hybrid detectors have improved this to $<1 \mathrm{~ns}$. On TAC/ADC based systems an upper limit to the detection efficiency is usually placed by the TCSPC electronics, which have typical dead-times in the order of $100 \mathrm{~ns}$. TDCs have improved this to $<1 \mathrm{~ns}$, albeit with considerable loss of timing resolution [51].

Today, many commercial setups for scanning FLIM use hybrid detectors combined with TAC/ADC electronics. Hybrid detectors combine the advantages of both PMTs and SPADs: they have high gain, short dead-time, and low timing jitter. TAC/ADC electronics, on the other hand, have excellent timing resolution and can achieve IRFs down to 20 ps. While the electronics deadtime is usually in the order of $100 \mathrm{~ns}$, the detection count rate is typically limited by the pile-up restrictions below $1 \mathrm{MHz}$.

It is possible to improve the overall image acquisition speed by using multiple detectors $[147,148]$ to reduce the dead-time and distribute the photons over the detectors e.g., by spectral dispersion. Multi-point scanning with SPAD arrays has also been demonstrated $[97,98]$ which enables real-time imaging of a FRET-based biosensor to study cell signaling, without trade-off between speed, noise and precision [149].

\subsection{Wide-Field FLIM}

In wide-field FLIM, the entire sample is illuminated to produce fluorescence, and the decay for each pixel is acquired in a parallel
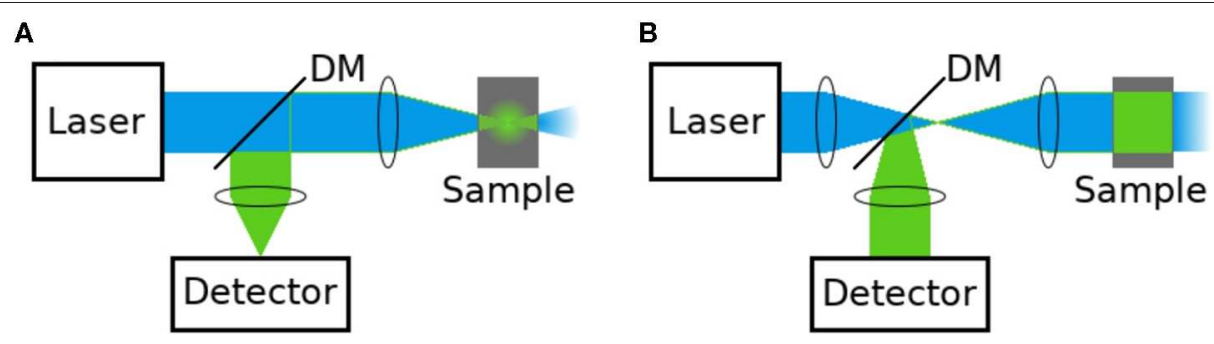

FIGURE 10 | Schematic diagram of the acquisition principle for (A) scanning and (B) wide-field FLIM. (A) In scanning FLIM, a focussed laser beam is raster-scanned over the image pixel by pixel to excite the fluorescence, and the photon arrival timing is performed with a point detector, separately in each pixel. (B) In wide-field FLIM, the entire sample is illuminated with a collimated excitation beam to excite the fluorescence, and the photon arrival timing is done with a position-sensitive detector. DM is dichroic mirror. 
manner using some kind of camera, i.e., a position-sensitive detector. There are many different methods to perform wide-field FLIM, including time-gating and frequency-domain methods which are not based on timing single photons and are out of scope of this review-for more information see, for example, [20, 150, $151]$. Image intensified fast cameras $[152,153]$, as well as a timestamping camera, TimepixCam $[154,155]$, have been used for timing single photons, but the photon timing resolution of these devices is limited from nanoseconds to microseconds, and thus they are more suitable for microsecond phosphorescence lifetime imaging rather than fast timing applications for nanosecond fluorescence decays. However, the latest TimepixCam version has $1.5 \mathrm{~ns}$ timing resolution [155] which would be suitable for measuring fluorescence decays of tens of nanoseconds.

For wide-field single photon timing on the picosecond time scale, MCPs and SPAD arrays are the most commonly used detectors. MCPs are a mature technology, and are used in many fields of science. Similar to PMTs, MCPs are capable of high timing resolution with IRFs down to 20 ps [79]. As they provide many channels, the position information is preserved during the signal amplification process. However, the MCPs themselves do not provide positional read-out, so they need to be combined with position read-out anodes [61, 156, 157]. Many different architectures have been designed [47], but usually the position read-out restricts the detection rate to a maximum of one photon per excitation pulse. Although advanced read-out architectures can improve this [158], overall this method does not offer significant improvement to the acquisition speed over scanning methods.

SPAD arrays are a more recent development in widefield single photon detection. Unlike MCPs where the position read-out often limits the detection rate to a maximum of 1 photon/excitation pulse, SPADs can count a photon/pixel/excitation pulse independently and therefore have potential for enormous count rates, and hence short acquisition times. The TDCs used in SPAD arrays can have an order of magnitude worse timing resolution than MCPs, but this is still acceptable for many FLIM applications where typical lifetimes are in the order of a few nanoseconds. The latest SPAD arrays can have fill factors of 50\% [99], a great improvement over the megaframe SPAD arrays with $1 \%$ fill factor $[97,98]$, but they generally do have some non-uniformity across the array. However, these specifications are continuously improving, as this technology is currently developing at a fast pace $[102,110]$.

Wide-field photon counting methods are especially useful for combining FLIM with microscopy techniques that usually require a wide-field detector, such as TIRF [159] or lightsheet [160] illumination methods. They are also indispensable in live cell imaging, where the uniformly distributed, extremely low light dose enables long term observation of sensitive specimens $[61,156,161,162]$.

\section{APPLICATIONS}

FLIM with fast timing has a vast range of applications in many different fields of science $[143,163]$. FLIM is often used for monitoring the microenvironment of living cells, such as $\mathrm{pH}$ [164], ion concentration $\left(\mathrm{Ca}^{2+}, \mathrm{Cl}^{-}, \mathrm{K}, \mathrm{Na}, \ldots\right)$ [165], viscosity $[166,167]$, temperature $[168,169]$, or oxygen levels $[170,171]$. FLIM is also the most precise methods for detecting FRET, which can be used for monitoring protein interaction, tension and folding [18]. A number of reviews and textbooks have delved into more details of the technique and its concepts, its implementations, applications and data analysis methods [20, 48, $49,51,151,172-181]$.
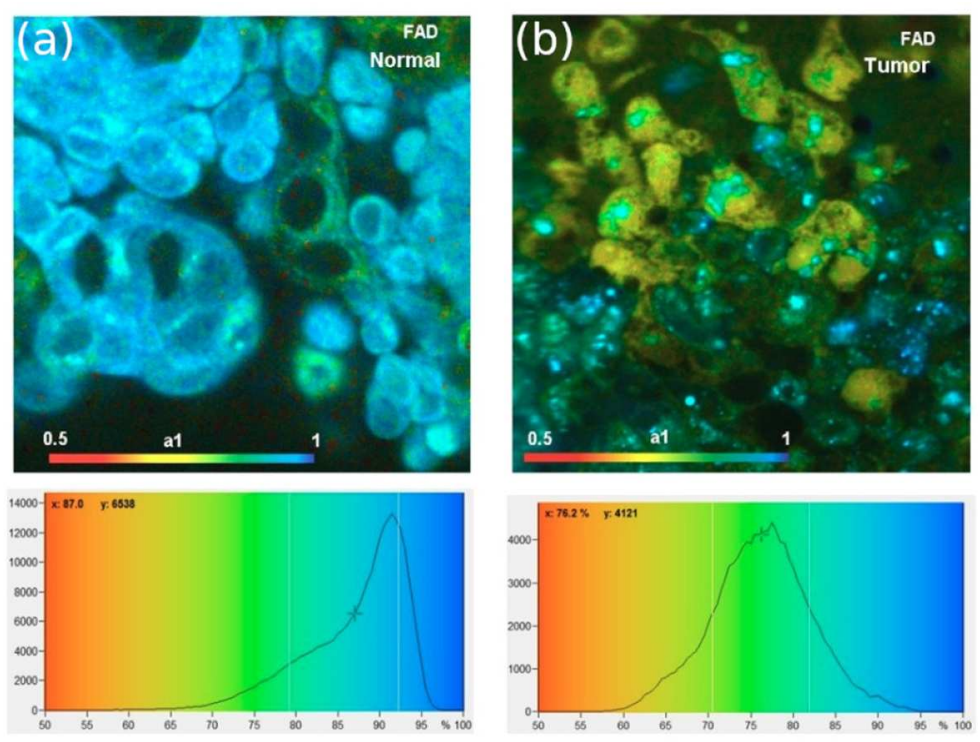

FIGURE 11 | Applications of fast scanning FLIM. (a,b) Metabolic state monitoring of cells with fast scanning FLIM. The images show the bound fraction of FAD, which decreases for cancer cells. Reproduced from Becker et al. [183]. 

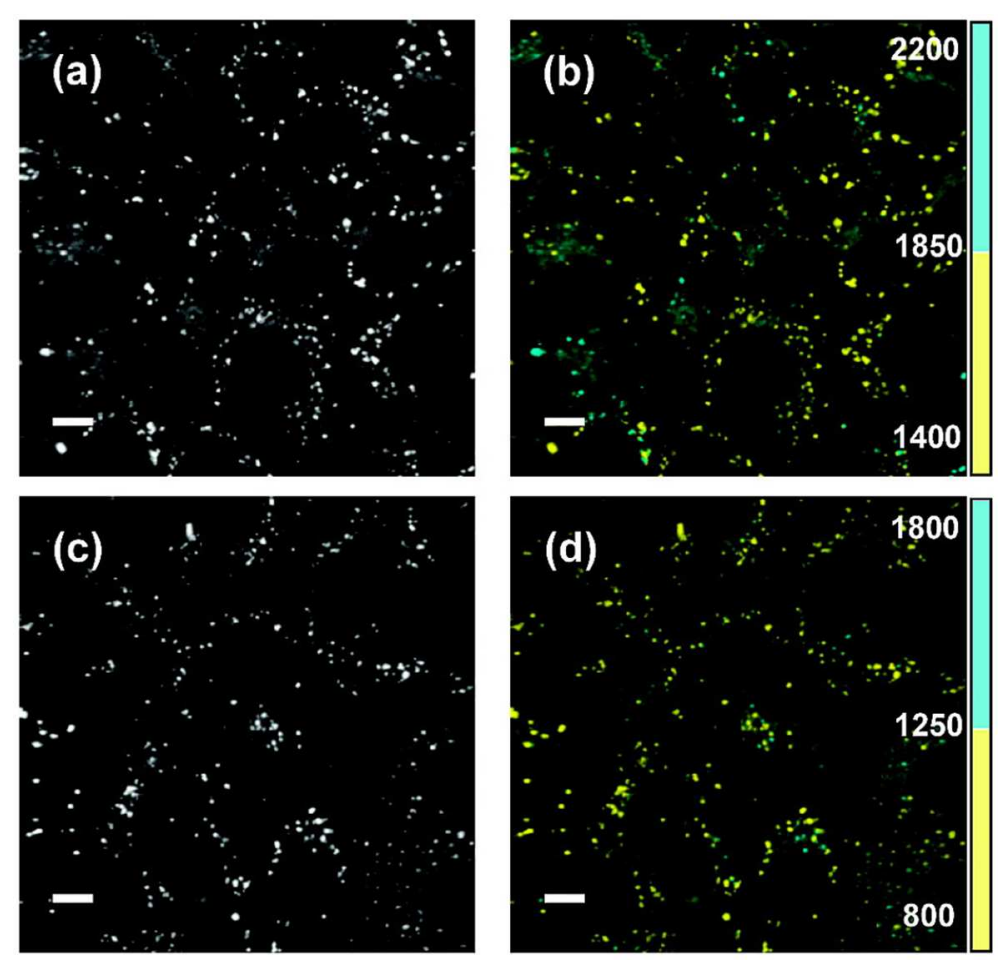

FIGURE 12 | FLIM viscosity measurement. Fluorescence intensity (a,c) and FLIM images (b,d) for live SK-OV-3 human ovarian carcinoma cells incubated with two different viscosity-sensitive dyes (bottom \& top). The fluorescence lifetimes are encoded in two discrete color scales, blue for a long lifetime range, indicating a higher viscosity, and yellow for a short lifetime range, indicating a lower viscosity. The lifetime range for the fluorescent molecular rotor dyes used in (b) is 1,400-1,850 ps in yellow and 1,850-2,200 ps in blue, and for the dye used in (d) 800-1,250 ps in yellow and 1,250-1,800 ps in blue. Scale bars: 10 $\mu$ m. Reproduced from [185].
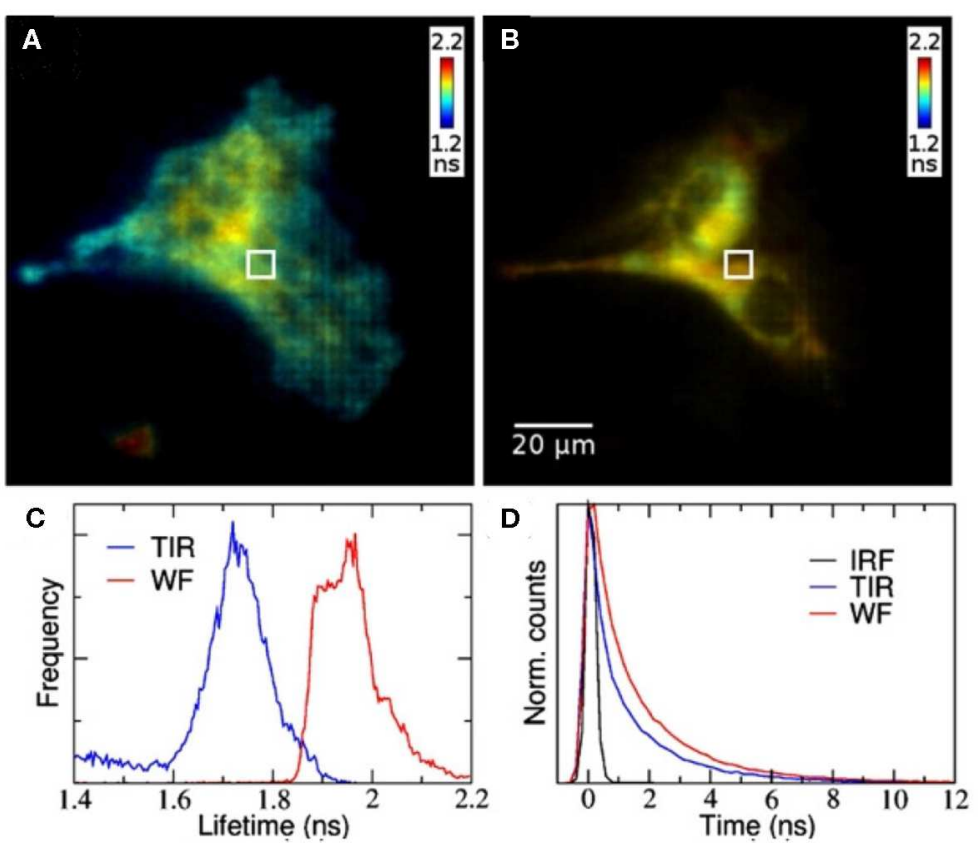

FIGURE 13 | Wide-field TCSPC-based FLIM with TIRF. (A) TIRF and (B) wide-field FLIM images of HeLa cells. (C) Fluorescence lifetime histograms and (D) representative fluorescence decays. The fluorescence lifetime difference between the images is due to selective excitation of the membrane-sensitive dye. Reproduced from Hirvonen et al. [159]. 

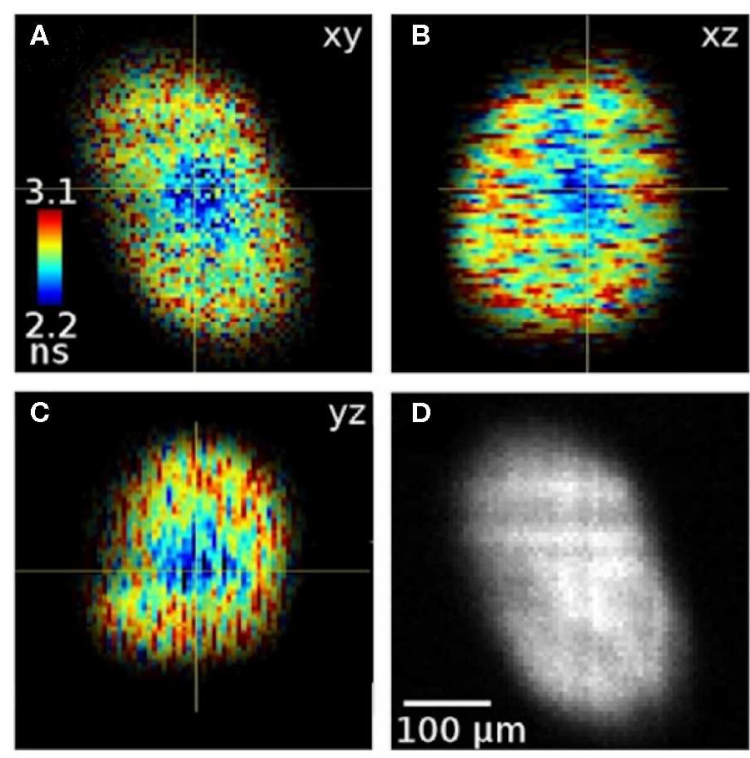
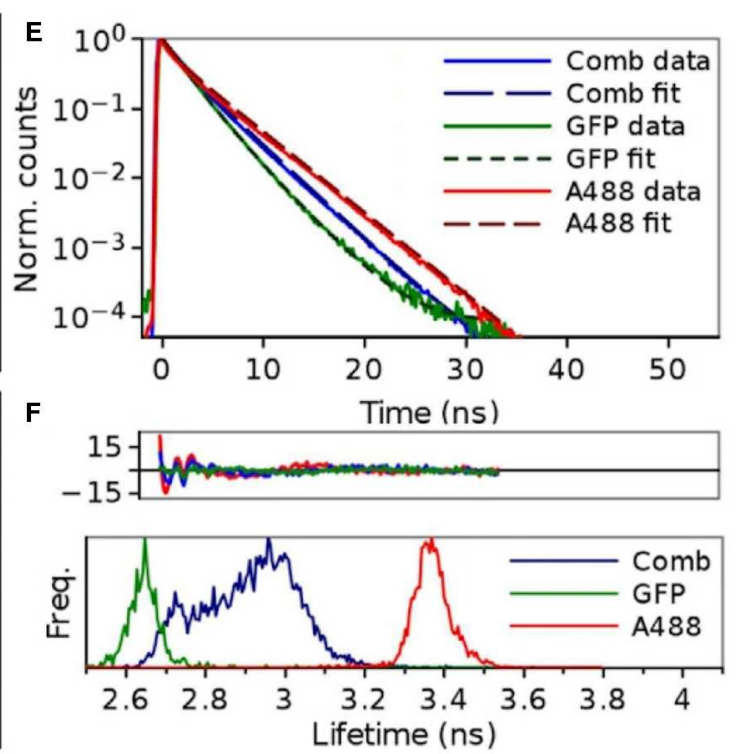

FIGURE 14 | TCSPC-based lightsheet FLIM images of cancer cell spheroids with two different fluorescent labels, one throughout the spheroid and one on the surface only. (A) xy FLIM image, with the color-encoded fluorescence lifetime contrast given by the color scale bar on the left, (B) xz FLIM image, (C) yz FLIM image, and (D) xy fluorescence intensity image, with a $100 \mu \mathrm{m}$ scale bar to indicate spatial dimensions. (E) Representative fluorescence decays of samples with one or both fluorescent labels on a semi-logarithmic plot. (F) Fluorescence lifetime histograms of samples with one or both fluorescent labels. Reproduced from Hirvonen et al. [160].

Typical fluorescence lifetimes are on the scale of a few nanoseconds which in principle can tolerate IRF widths of a few hundred ps. However, fast ps timing accuracy helps in detecting subtle changes in the microenvironment of living cells, or changes in FRET levels, especially in applications where the overall imaging speed is also a critical parameter [149].

Commercial scanning systems are now available that routinely achieve both fast photon timing accuracy, and fast overall image acquisition times. A confocal FLIM system has been reported with IRF FWHM $<25$ ps, which achieves count rates of up to $20 \mathrm{MHz}$ [182]. The system uses a single detector but routes the photons into four separate timing channels, thereby shortening the TCSPC electronics dead-time. This system can obtain a 256 $\times 256$ pixel FLIM image in about $0.5 \mathrm{~s}$.

This type of system has been used, for example, for monitoring the metabolic state in living cells [183], see Figure 11. This approach allows for an optical biopsy to be obtained, to assess cells for possible signs of disease, without employing mechanical methods for tissue removal or chemical methods for analysis. This so-called autofluorescence FLIM (because the sample does not need to be stained with fluorescence dyes, the intrinsic fluorescence of cells is used) is used for clinical diagnostics of skin [184]. Two lasers were multiplexed to alternatingly excite the endogenous fluorophores $\mathrm{NAD}(\mathrm{P}) \mathrm{H}$ and FAD which provide a read-out of the metabolic state, detected in two FLIM channels. Information on the metabolic state was derived from the fluorescence decays of $\mathrm{NAD}(\mathrm{P}) \mathrm{H}$ and $\mathrm{FAD}$, which both have a fast few 100 ps and a slow ns scale component corresponding to bound and unbound fractions. A fast FLIM system is able to detect subtle changes in the component amplitude ratios and therefore changes in the metabolic state of the cells.

FLIM has also been used for monitoring viscosity in living cells using viscosity-sensitive fluorescent molecular rotor dyes [185], see Figure 12. Viscosity is the resistance of a fluid to flow, and is a key parameter determining chemical or biochemical reaction rates. Traditionally, bulk viscosity is measured by mechanical methods, but the use of fluorescent molecular rotors allows optical viscosity measurements. For this approach, only a very small sample is needed, and in combination with fluorescence microscopy and FLIM allows mapping viscosity on a micrometer scale, for example in living cells and their organelles [186].

The FLIM examples in Figures 11 and $\mathbf{1 2}$ are based on raster scanning a single point across the image, and performing TCSPC in each pixel separately, and, depending on the circumstances, it typically takes minutes to acquire a sufficient amount of photons for fluorescence decay analysis. The imaging speed can be improved by multi-point scanning. An $8 \times 8$ beamlet array was used to illuminate a sample, with a $32 \times 32$ pixel SPAD array detecting the emission from the spots excited by these beamlets with 55 ps timing resolution. Each beamlet scanned a $32 \times 32$ pixel area, producing a final image with $256 \times 256$ pixels. The original system had image acquisition time of $10 \mathrm{~s} /$ optical slice, the authors were able to detect FRET and calculate differences in cell biochemistry between sample $[97,98]$. An improved version was able to image four optical planes simultaneously with $4 \times$ 4 beamlets in each optical plane, improving overall acquisition speed by a factor of 64 compared to conventional scanning FLIM [187]. With a more powerful laser, the generation of $32 \times 32$ 
beamlets could allow $8 \times 8$ beamlet acquisition of 16 individual planes simultaneously.

In wide-field TCSPC imaging, MCPs provide excellent timing resolution, but they need to be combined with a positionsensitive read-out [61, 156, 157]. Recently an MCP detector with picosecond timing resolution was combined with a delay line anode for position-sensitive read-out, and this detector combined with fluorescence microscopy techniques that are difficult to implement with scanning technologies [188].

TIRF microscopy is a method where an evanescent wave, created by reflection of an excitation beam via total internal reflection on a microscope cover slip, penetrates a few hundred nanometers into the sample. This technique is typically used to excite dyes or fluorescent proteins in or near membranes close to the coverslip, without creating background fluorescence from regions inside the cell. TIRF images are usually recorded with a camera, rather than raster scanning. Figure 13 shows a comparison of TIRF (left) and wide-field (right) fluorescence lifetime images of HeLa cells where the lifetime difference between the images is due to a membrane-sensitive dye [159]. The TCSPC-based wide-field FLIM approach for TIRF relies on the crossed delay line anode detector, which provides spatial and timing information for each detected photon.

Lightsheet fluorescence microscopy is another microscopy approach which is typically carried out using a camera. Figure 14 shows lightsheet FLIM images of cancer cell spheroids with two different fluorescent labels, one throughout the spheroid and one on the surface only [160]. Again, without a positionsensitive detector with picosecond time resolution, TCSPC-based lightsheet FLIM is hard to perform.

\section{CONCLUSIONS}

FLIM is a widely used imaging technique in the life sciences which allows not only the localization of fluorophores, but also the monitoring of their microenvironment and their

\section{REFERENCES}

1. Bradley J. IV. A letter from the Reverend Mr. James Bradley Savilian Professor of Astronomy at Oxford, and F. R. S. to Dr. Edmond Halley Astronom. Reg. \&c. giving an Account of a new discovered Motion of the Fix'd Stars. Philos T R Soc. (1728) 35:637-61. doi: 10.1098/rstl.1727.0064

2. Maxwell JC. VIII. A dynamical theory of the electromagnetic field. Philos T R Soc. (1865) 155:459-512. doi: 10.1098/rstl.1865.0008

3. Wheatstone C, Faraday M. An account of some experiments to measure the velocity of electricity and the duration of electric light. Philos T R Soc. (1834) 124:583-91. doi: 10.1098/rstl.1834.0031

4. Einstein A. Über einen die Erzeugung und Verwandlung des Lichtes betreffenden heuristischen Gesichtspunkt. Ann Phys. (1905) 322:132-48. doi: 10.1002/andp.190532 20607

5. Lewis GN. The conservation of photons. Nature. (1926) 118:874-5. doi: $10.1038 / 118874 \mathrm{a} 0$

6. Roychoudhuri C, Kracklauer AF, Creath K. The Nature of Light: What Is a Photon? Boca Raton, FL: CRC Press (2008).

7. Hertz H. Ueber einen Einfluss des ultravioletten Lichtes auf die electrische Entladung. Ann Phys. (1887) 267:983-1000. doi: 10.1002/andp.18872670827 interaction, typically via their nanosecond fluorescence decay. Photon counting methods allow the measurement of photon arrival time with picosecond time resolution. Both detectors and electronics play a critical role in fast timing of photons. From the detector side, transit time spread in the signal amplification process is a critical parameter for fast timing, whereas low dead-time and high detection efficiency are also important in fluorescence applications. For timing, the mature TAC/ADC technology can now achieve timing accuracy of a few ps, and is the most precise technology to use for fast timing applications. TDCs are a newer technology, whose timing capabilities are still limited to tens of ps by clock speeds, but they are easier to produce, more robust against environmental variations and easier to upscale, for example, to use in megapixel detector array technology for SPADs and SiPMs.

For fluorescence measurements, TCSPC has the best signal-to-noise ratio of the standard time-resolved imaging methods, and is accurate enough to allow multiexponential fluorescence decay fitting. The extremely low illumination intensity, distributed evenly over the field of view, is beneficial especially in life science applications where it allows long-term monitoring of living cells and organisms. The development of fast TCSPC methods will also benefit fields where single photon or particle time-of-flight measurements are required, for example LIDAR, neutron imaging ion velocity mapping or photon correlation techniques.

\section{AUTHOR CONTRIBUTIONS}

All authors listed have made a substantial, direct and intellectual contribution to the work, and approved it for publication.

\section{FUNDING}

KS acknowledges funding from BBSRC (BB/R004803/1).
8. Austin L, Starke H. Ueber die Reflexion der Kathodenstrahlen und eine damit verbundene neue Erscheinung secundärer Emission. Ann Phys. (1902) 314:271-292. doi: 10.1002/andp.19023141003

9. Bothe W, Kolhörster W. Das Wesen der Höhenstrahlung. Z Phys. (1929) 56:751-77. doi: 10.1007/BF01340137

10. Rossi B. Method of registering multiple simultaneous impulses of several Geiger's counters. Nature. (1930) 125:636-6. doi: 10.1038/125636a0

11. Rossi B, Nereson N. Experimental determination of the disintegration curve of mesotrons. Phys Rev. (1942) 62:417-22. doi: 10.1103/PhysRev. 62.417

12. Bell RE, Graham RL, Petch HE. Design and use of a coincidence circuit of short resolving time. Can J Phys. (1952) 30:35-52. doi: 10.1139/p52-004

13. Lundby A. Scintillation decay times. Phys Rev. (1950) 80:477. doi: 10.1103/PhysRev.80.477

14. Bollinger LM, Thomas GE. Measurement of the time dependence of scintillation intensity by a delayed-coincidence method. Rev Sci Instrum. (1961) 32:1044. doi: 10.1063/1.1717610

15. O'Connor DV, Phillips D. Time-Correlated Single-Photon Counting. New York, NY: Academic Press (1984).

16. Lakowicz JR. Principles of Fluorescence Spectroscopy. 3rd edn. New York, NY: Springer (2006). doi: 10.1007/978-0-387-46312-4 
17. Toptygin D. Effects of the solvent refractive index and its dispersion on the radiative decay rate and extinction coefficient of a fluorescent solute. $J$ Fluoresc. (2003) 13:201-19. doi: 10.1023/A:1025033731377

18. Algar WR, Hildebrandt N, Vogel SS, Medintz IL. FRET as a biomolecular research tool - understanding its potential while avoiding pitfalls. Nat Methods. (2019) 16:815-29. doi: 10.1038/s41592-019-0530-8

19. Esposito A. How many photons are needed for FRET imaging? Biomed Opt Express. (2020) 11:1186-1202. doi: 10.1364/BOE.379305

20. Gadella TWJ, editor. FRET and FLIM Techniques. Amsterdam, Boston, MA: Elsevier (2009).

21. Schuyler R, Isenberg I. A monophoton fluorometer with energy discrimination. Rev Sci Instrum. (1971) 42:813-7. doi: 10.1063/1.1685237

22. Bachrach RZ. A photon counting apparatus for kinetic and spectral measurements. Rev Sci Instrum. (1972) 43:734. doi: 10.1063/1.1685743

23. Binkert T, Tschanz HP, Zinsli PE. The measurement of fluorescence decay curves with the single-photon counting method and the evaluation of rate parameters. J Lumin. (1972) 5:187-217. doi: 10.1016/0022-2313(72)90041-5

24. Lewis C, Ware WR, Doemeny LJ, Nemzek TL. The measurement of shortlived fluorescence decay using the single photon counting method. Rev Sci Instrum. (1973) 44:107-14. doi: 10.1063/1.1686062

25. Phillips D. A lifetime in photochemistry; some ultrafast measurements on singlet states. Philos R Soc Lond A Mat. (2016) 472:20160102. doi: 10.1098/rspa.2016.0102

26. Previte MJR, Zhou C, Kellinger M, Pantoja R, Chen CY, Shi J, et al. DNA sequencing using polymerase substrate-binding kinetics. Nat Commun. (2015) 6:5936. doi: 10.1038/ncomms6936

27. Hemmati H. Interplanetary laser communications. Opt Photon News. (2007) 18:22-7. doi: 10.1364/OPN.18.11.000022

28. Buller GS, Wallace AM. Ranging and three-dimensional imaging using timecorrelated single-photon counting and point-by-point acquisition. IEEE J Sel Top Quant. (2007) 13:1006-15. doi: 10.1109/JSTQE.2007.902850

29. McCarthy A, Collins RJ, Krichel NJ, Fernández V, Wallace AM, Buller GS. Long-range time-of-flight scanning sensor based on high-speed time-correlated single-photon counting. Appl Optics. (2009) 48:6241. doi: 10.1364/AO.48.006241

30. Turgeman L, Fixler D. Time-averaged fluorescence intensity analysis in fluorescence fluctuation polarization sensitive experiments. Biomed Opt Express. (2013) 4:868-84. doi: 10.1364/BOE.4.000868

31. Schmidt FEW, Fry ME, Hillman EMC, Hebden JC, Delpy DT. A 32-channel time-resolved instrument for medical optical tomography. Rev Sci Instrum. (2000) 71:256-65. doi: 10.1063/1.1150191

32. Buller GS, Collins RJ. Single-photon generation and detection. Meas Sci Technol. (2010) 21:012002. doi: 10.1088/0957-0233/21/1/012002

33. Hadfield RH. Single-photon detectors for optical quantum information applications. Nat Photonics. (2009) 3:696-705. doi: 10.1038/nphoton.2009.230

34. Eisaman MD, Fan J, Migdall A, Polyakov SV. Single-photon sources and detectors. Rev Sci Instrum. (2011) 82:071101. doi: 10.1063/1.3610677

35. Fraser GW, Pearson JF. The direct detection of thermal neutrons by imaging microchannel plate detectors. Nuclear Instrum Methods A. (1990) 293:569-74. doi: 10.1016/0168-9002(90)90325-Z

36. Tremsin AS, McPhate JB, Vallerga JV, Siegmund OHW, Feller WB, Lehmann E, et al. Neutron radiography with sub-15 micron resolution through event centroiding. Nuclear Instrum Methods A. (2012) 688:32-40. doi: 10.1016/j.nima.2012.06.005

37. Vallance C, Brouard M, Lauer A, Slater CS, Halford E, Winter B, et al. Fast sensors for time-of-flight imaging applications. Phys Chem Chem Phys. (2014) 16:383-95. doi: 10.1039/C3CP53183J

38. Dinu L, Eppink ATJB, Rosca-Pruna F, Offerhaus HL, van der Zande WJ, Vrakking MJJ. Application of a time-resolved event counting technique in velocity map imaging. Rev Sci Instrum. (2002) 73:4206-4213. doi: $10.1063 / 1.1520732$

39. Strasser D, Urbain X, Pedersen HB, Altstein N, Heber O, Wester $\mathrm{R}$, et al. An innovative approach to multiparticle three-dimensional imaging. Rev Sci Instrum. (2000) 71:3092-3098. doi: 10.1063/1.130 5514

40. Seitz P, Theuwissen AJP. Single Photon Imaging. Heidelberg: Springer (2011). doi: 10.1007/978-3-642-18443-7
41. Amos WB, White JG. How the confocal laser scanning microscope entered biological research. Biol Cell. (2003) 95:335-42. doi: 10.1016/S0248-4900(03)00078-9

42. Bugiel I, König K, Wabnitz H. Investigation of cells by fluorescence laser scanning microscopy with subnanosecond time resolution. Lasers Life Sci. (1989) 3:47-53.

43. Morgan CG, Mitchell AC, Murray JG. Nanosecond time-resolved fluorescence microscopy: principles and practice. Proc $R$ Microscop Soc. (1990) 1:463-66.

44. Wang XF, Periasamy A, Herman B, Coleman DM. Fluorescence lifetime imaging microscopy (FLIM): instrumentation and applications. Crit Rev Anal Chem. (1992) 23:369-95. doi: 10.1080/10408349208051651

45. Gadella TWJ, Jovin TM, Clegg RM. Fluorescence lifetime imaging microscopy (FLIM): spatial resolution of microstructures on the nanosecond time scale. Biophys Chem. (1993) 48:221-39. doi: 10.1016/0301-4622(93)85012-7

46. Becker W, Bergmann A, Hink MA, König K, Benndorf K, Biskup C. Fluorescence lifetime imaging by time-correlated single-photon counting. Microsc Res Tech. (2004) 63:58-66. doi: 10.1002/jemt.10421

47. Hirvonen LM, Suhling K. Wide-field TCSPC: methods and applications. Meas Sci Technol. (2017) 28:012003. doi: 10.1088/1361-6501/28/1/012003

48. Becker W. Advanced Time-Correlated Single Photon Counting Techniques. Berlin; Heidelberg: Springer (2005). doi: 10.1007/3-540-28882-1

49. Becker W. Advanced Time-Correlated Single Photon Counting Applications. Vol. 111. Cham: Springer International Publishing (2015). doi: 10.1007/978-3-319-14929-5

50. Kalisz J. Review of methods for time interval measurements with picosecond resolution. Meterologia. (2003) 41:17-32. doi: 10.1088/0026-1394/41/1/004

51. Liu X, Lin D, Becker W, Niu J, Yu B, Liu L, et al. Fast fluorescence lifetime imaging techniques: a review on challenge and development. J Innov Opt Heal Sci. (2019) 12:1930003. doi: 10.1142/S1793545819 300039

52. Birch DJS, Imhof RE. Time-Domain Fluorescence Spectroscopy Using TimeCorrelated Single-Photon Counting. In: Lakowicz JR, editor. Topics in Fluorescence Spectroscopy: Techniques. Boston, MA: Springer (1999). p. 1-95. doi: 10.1007/0-306-47057-8_1

53. Wahl M. Modern TCSPC electronics: principles and acquisition modes. In: Kapusta P, Wahl M, Erdmann R, editors. Advanced Photon Counting. Springer Series on Fluorescence (Methods and Applications). Cham: Springer (2014). p. 15.

54. Iams H, Salzberg B. The secondary emission phototube. Proc IRE. (1935) 23:55-64. doi: 10.1109/JRPROC.1935.227243

55. Kubetsky LA. Multiple amplifier. Proc IRE. (1937) 25:421-33. doi: 10.1109/JRPROC.1937.229045

56. Zworykin VK, Morton GA, Malter L. The secondary emission multiplier-a new electronic device. Proc IRE. (1936) 24:351-75. doi: 10.1109/JRPROC.1936.226435

57. Allen JS. The detection of single positive ions, electrons and photons by a secondary electron multiplier. Phys Rev. (1939) 55:966-71. doi: 10.1103/PhysRev.55.966

58. Candy BH. Photomultiplier characteristics and practice relevant to photon counting. Rev Sci Instrum. (1985) 56:183-93. doi: 10.1063/1.1138327

59. Hungerford G, Birch DJS. Single-photon timing detectors for fluorescence lifetime spectroscopy. Meas Sci Technol. (1996) 7:121. doi: 10.1088/0957-0233/7/2/002

60. Michalet X, Siegmund OH, Vallerga JV, Jelinsky P, Millaud JE, Weiss S. Detectors for single-molecule fluorescence imaging and spectroscopy. J Mod Opt. (2007) 54:239. doi: 10.1080/09500340600769067

61. Michalet X, Colyer RA, Scalia G, Ingargiola A, Lin R, Millaud JE, et al. Development of new photon-counting detectors for singlemolecule fluorescence microscopy. Philos T R Soc B. (2013) 368:1611. doi: $10.1098 /$ rstb.2012.0035

62. van Meurs B, van der Werf R. A circuit for rejecting multiple events detected during a timing cycle in single photon counting experiments. J Phys E Sci Instrum. (1976) 9:437-8. doi: 10.1088/0022-3735/9/6/005

63. Fukuda S, Fukuda Y, Hayakawa T, Ichihara E, Ishitsuka M, Itow Y, et al. The Super-Kamiokande detector. Nuclear Instrum Methods A. (2003) 501:418-462. doi: 10.1016/S0168-9002(03)00425-X 
64. Bird DK, Eliceiri KW, Fan CH, White JG. Simultaneous two-photon spectral and lifetime fluorescence microscopy. Appl Opt. (2004) 43:5173-82. doi: 10.1364/AO.43.005173

65. Biskup C, Zimmer T, Kelbauskas L, Hoffmann B, Klocker N, Becker W, et al. Multi-dimensional fluorescence lifetime and FRET measurements. Microsc Res Tech. (2007) 70:442-51. doi: 10.1002/jemt.20431

66. Farnsworth PT. Electron Multiplier (1934). US Patent 1969399.

67. Wiley WC, Hendee CF. Electron multipliers utilizing continuous strip surfaces. IRE T Nuclear Sci. (1962) 9:103-6. doi: 10.1109/TNS2.1962.4315981

68. Goodrich GW, Wiley WC. Continuous channel electron multiplier. Rev Sci Instrum. (1962) 33:761. doi: 10.1063/1.1717958

69. Lampton M. The microchannel image intensifier. Sci Am. (1981) 245:62-71. doi: 10.1038/scientificamerican1181-62

70. Siegmund OHW, Vallerga JV, Tremsin AS, McPhate J, Michalet X, Colyer RA, et al. Microchannel plate imaging photon counters for ultraviolet through NIR detection with high time resolution. Proc SPIE. (2011) 8033:80330V-12. doi: 10.1117/12.884271

71. Siegmund OHW. High-performance microchannel plate detectors for UV/visible astronomy. Nuclear Instrum Methods A. (2004) 525:12-6. doi: 10.1016/j.nima.2004.03.018

72. Wang X, Setru SU, Xie J, Mane A, Demarteau M, Wagner R. Imaging of large-area microchannel plates using phosphor screens. J Instrum. (2014) 9:P11011. doi: 10.1088/1748-0221/9/11/P11011

73. Boksenberg A. Advances in detectors for astronomical spectroscopy. Philos T R Soc S A. (1982) 307:531-48. doi: 10.1098/rsta.1982.0128

74. Kröger HW, Schmidt GK, Pailer N. Faint object camera: European contribution to the Hubble Space Telescope. Acta Astronaut. (1992) 26:827-34. doi: 10.1016/0094-5765(92)90063-O

75. Mason KO, Breeveld A, Much R, Carter M, Cordova FA, Cropper MS, et al. The XMM-Newton optical/UV monitor telescope. Astron Astrophys. (2001) 365:L36-44. doi: 10.1051/0004-6361:20000044

76. Roming PW, Kennedy TE, Mason KO, et al. The swift ultra-violet/optical telescope. Space Sci Rev. (2005) 120:95-42. doi: 10.1007/s11214-005-5095-4

77. Siegmund OHW, Welsh BY, Martin C, et al. The GALEX mission and detectors. Proc SPIE. (2004) 5488:13-24. doi: 10.1117/12.561488

78. Conti L, Barnstedt J, Hanke L, Kalkuhl C, Kappelmann N, Rauch T, et al. MCP detector development for UV space missions. Astrophys Space Sci. (2018) 363:63. doi: 10.1007/s10509-018-3283-4

79. Tremsin AS, Vallerga JV, Siegmund OHW. Overview of spatial and timing resolution of event counting detectors with Microchannel Plates. Nuclear Instrum Methods A. (2020) 949:162768. doi: 10.1016/j.nima.2019.162768

80. Franco A, Geissbühler J, Wyrsch N, Ballif C. Fabrication and characterization of monolithically integrated microchannel plates based on amorphous silicon. Sci Rep. (2014) 4:4597. doi: 10.1038/srep04597

81. Ertley CD, Siegmund OHW, Hull J, Tremsin A, O'Mahony A, Craven CA, et al. Microchannel plate imaging detectors for high dynamic range applications. IEEE T Nuclear Sci. (2017) 64:1774-80. doi: 10.1109/TNS.2017.2652222

82. Louis T, Schatz GH, Klein-Bölting P, Holzwarth AR, Ripamonti G, Cova S. Performance comparison of a single-photon avalanche diode with a microchannel-plate photomultiplier in time-correlated single-photon counting. Rev Sci Instrum. (1988) 59:1148-52. doi: 10.1063/1.1140258

83. Ceccarelli F, Acconcia G, Gulinatti A, Ghioni M, Rech I. Fully integrated active quenching circuit driving custom-technology SPADs with 6.2-ns dead time. IEEE Photon Tech. (2019) 31:102-5. doi: 10.1109/LPT.2018.28 84740

84. Charbon E. Single-photon imaging in complementary metal oxide semiconductor processes. Philos T R Soc A. (2014) 372:20130100. doi: $10.1098 /$ rsta.2013.0100

85. Charbon E, Fishburn M, Walker R, Henderson RK, Niclass C. SPAD-based sensors. In: Remondino F, Stoppa D, editors. TOF RangeImaging Cameras. Berlin; Heidelberg: Springer (2013). p. 11-38. doi: 10.1007/978-3-642-27523-4_2

86. Colyer RA, Scalia G, Rech I, Gulinatti A, Ghioni M, Cova S, et al. Highthroughput FCS using an LCOS spatial light modulator and an $8 \times 1$ SPAD array. Biomed Opt Express. (2010) 1:1408-31. doi: 10.1364/BOE.1.0 01408
87. Cuccato A, Antonioli S, Crotti M, Labanca I, Gulinatti A, Rech I, et al. Complete and compact 32-channel system for time-correlated single-photon counting measurements. IEEE Photon J. (2013) 5:6801514. doi: 10.1109/JPHOT.2013.2284250

88. Krstajić N, Levitt J, Poland S, Ameer-Beg S, Henderson R. 256 x 2 SPAD line sensor for time resolved fluorescence spectroscopy. Opt Express. (2015) 23:5653-69. doi: 10.1364/OE.23.005653

89. Burri S, Homulle H, Bruschini C, Charbon E. LinoSPAD: a timeresolved 256x1 CMOS SPAD line sensor system featuring 64 FPGAbased TDC channels running at up to 8.5 giga-events per second. In: Berghmans F, Mignani AG, editors. Optical Sensing Detection IV. Vol. 9899. International Society for Optics and Photonics. Proc SPIE. (2016). p. 57-66. doi: 10.1117/12.2227564

90. Kufcsák A, Erdogan A, Walker R, Ehrlich K, Tanner M, Megia-Fernandez A, et al. Time-resolved spectroscopy at 19,000 lines per second using a CMOS SPAD line array enables advanced biophotonics applications. Opt Express. (2017) 25:11103-23. doi: 10.1364/OE.25.011103

91. Erdogan AT, Walker R, Finlayson N, Krstajić N, Williams G, Girkin J, et al. A CMOS SPAD line sensor with per-pixel histogramming TDC for time-resolved multispectral imaging. IEEE J Solid Circ. (2019) 54:1705-19. doi: 10.1109/JSSC.2019.2894355

92. Veerappan C, Richardson J, Walker R, Li D, Fishburn MW, Maruyama $\mathrm{Y}$, et al. A 160-128 single-photon image sensor with on-pixel 55ps 10b time-to-digital converter. In: 2011 IEEE International Solid-State Circuits Conference (2011). p. 312-4. doi: 10.1109/ISSCC.2011.5746333

93. Field RM, Realov S, Shepard KL. A 100 fps, time-correlated single-photoncounting-based fluorescence-lifetime imager in $130 \mathrm{~nm}$ CMOS. IEEE J Solid Circ. (2014) 49:867-80. doi: 10.1109/JSSC.2013.2293777

94. Tamborini D, Buttafava M, Ruggeri A, Zappa F. Compact, lowpower and fully reconfigurable 10 ps resolution, $160 \mu$ range, timeresolved single-photon counting system. IEEE Sens J. (2016) 16:3827-33. doi: 10.1109/JSEN.2016.2535403

95. Krstajić N, Poland S, Levitt J, Walker R, Erdogan A, Ameer-Beg S, et al. 0.5 billion events per second time correlated single photon counting using CMOS SPAD arrays. Opt Lett. (2015) 40:4305-8. doi: 10.1364/OL.40.004305

96. Ulku A, Ardelean A, Antolovic M, Weiss S, Charbon E, Bruschini C, et al. Wide-field time-gated SPAD imager for phasor-based FLIM applications. Methods Appl Fluoresc. (2020) 8:024002. doi: 10.1088/2050-6120/ab6ed7

97. Poland SP, Krstajić N, Coelho S, Tyndall D, Walker RJ, Devauges V, et al. Time-resolved multifocal multiphoton microscope for high speed FRET imaging in vivo. Opt Lett. (2014) 39:6013-6. doi: 10.1364/OL.39.006013

98. Poland SP, Krstajić N, Monypenny J, Coelho S, Tyndall D, Walker RJ, et al. A high speed multifocal multiphoton fluorescence lifetime imaging microscope for live-cell FRET imaging. Biomed Opt Express. (2015) 6:277-96. doi: 10.1364/BOE.6.000277

99. Hutchings SW, Johnston N, Gyongy I, Abbas TA, Dutton NAW, Tyler $\mathrm{M}$, et al. A Reconfigurable 3-D-stacked SPAD imager with in-pixel histogramming for flash LIDAR or high-speed time-of-flight imaging. IEEE J Solid Circ. (2019) 54:2947-56. doi: 10.1109/JSSC.2019.2939083

100. Gyongy I, Calder N, Davies A, Dutton NAW, Duncan RR, Rickman C, et al. A $256 \times 256,100-\mathrm{kfps}, 61$

101. Parmesan L, Dutton N, Krstajic N, Calder N, Holmes A, Grant LA, et al. A 256 × 256 SPAD array with in-pixel Time to Amplitude Conversion for Fluorescence Lifetime Imaging Microscopy. In: Frontiers in Optics. OSA; 2015.

102. Bruschini C, Homulle H, Antolovic IM, Burri S, Charbon E. Single-photon SPAD imagers in biophotonics: review and outlook. Light Sci Appl. (2019) 8:87. doi: 10.1038/s41377-019-0191-5

103. Acerbi F, Gundacker S. Understanding and simulating SiPMs. Nuclear Instrum Methods A. (2019) 926:16-35. doi: 10.1016/j.nima.2018.11.118

104. Klanner R. Characterisation of SiPMs. Nuclear Instrum Methods A. (2019) 926:36-56. doi: 10.1016/j.nima.2018.11.083

105. Piemonte C, Gola A. Overview on the main parameters and technology of modern Silicon Photomultipliers. Nuclear Instrum Methods A. (2019) 926:2-15. doi: 10.1016/j.nima.2018.11.119

106. Martinenghi E, Di Sieno L, Contini D, Sanzaro M, Pifferi A, Dalla Mora A. Time-resolved single-photon detection module based on silicon 
photomultiplier: a novel building block for time-correlated measurement systems. Rev Sci Instrum. (2016) 87:073101. doi: 10.1063/1.4954968

107. Rech I, Ingargiola A, Spinelli R, Labanca I, Marangoni S, Ghioni M, et al. Optical crosstalk in single photon avalanche diode arrays: a new complete model. Opt Express. (2008) 16:8381-94. doi: 10.1364/OE.16.008381

108. Nagy F, Mazzillo M, Renna L, Valvo G, Sanfilippo D, Carbone B, et al. Afterpulse and delayed crosstalk analysis on a STMicroelectronics silicon photomultiplier. Nuclear Instrum Methods A. (2014) 759:44-49. doi: 10.1016/j.nima.2014.04.045

109. Xu H, Pancheri L, Braga LHC, Betta GFD, Stoppa D. Crosstalk characterization of single-photon avalanche diode (SPAD) arrays in CMOS 150nm technology. Proc Eng. (2014) 87:1270-3. doi: 10.1016/j.proeng.2014.11.417

110. Henderson RK, Johnston N, Mattioli Della Rocca F, Chen H, Li DDU, Hungerford G, et al. A $192 \times 128$ time correlated SPAD image sensor in 40-nm CMOS technology. IEEE J Solid Circ. (2019) 54:1907-16. doi: 10.1109/JSSC.2019.2905163

111. Caccia M, Nardo L, Santoro R, Schaffhauser D. Silicon photomultipliers and SPAD imagers in biophotonics: advances and perspectives. Nuclear Instrum Methods A. (2019) 926:101-17. doi: 10.1016/j.nima.2018.10.204

112. Li DU, Bonnist E, Renshaw D, Henderson R. On-chip, time-correlated, fluorescence lifetime extraction algorithms and error analysis. J Opt Soc Am A. (2008) 25:1190-8. doi: 10.1364/JOSAA.25.001190

113. Li DU, Arlt J, Richardson J, Walker R, Buts A, Stoppa D, et al. Realtime fluorescence lifetime imaging system with a $32 \times 320.13 \mu \mathrm{m}$ CMOS low dark-count single-photon avalanche diode array. Opt Express. (2010) 18:10257-69. doi: 10.1364/OE.18.010257

114. Poland SP, Erdogan AT, Krstajić N, Levitt J, Devauges V, Walker RJ, et al. New high-speed centre of mass method incorporating background subtraction for accurate determination of fluorescence lifetime. Opt Express. (2016) 24:6899-915. doi: 10.1364/OE.24.006899

115. Hirvonen LM, Jiggins S, Sergent N, Zanda G, Suhling K. Photon counting imaging with an electron-bombarded CCD: towards a parallel-processing photoelectronic time-to-amplitude converter. Rev Sci Instrum. (2014) 85:123102. doi: $10.1063 / 1.4901935$

116. Hirvonen L, Suhling K. Photon counting imaging with an electron-bombarded pixel image sensor. Sensors. (2016) $16: 617$. doi: $10.3390 / \mathrm{s} 16050617$

117. Becker W, Su B, Holub O, Weisshart K. FLIM and FCS detection in laser-scanning microscopes: increased efficiency by GaAsP hybrid detectors. Microsc Res Tech. (2011) 74:804-11. doi: 10.1002/jemt.20959

118. Michalet X, Cheng A, Antelman J, Suyama M, Arisaka K, Weiss S. Hybrid photodetector for single-molecule spectroscopy and microscopy. Proc SPIE. (2008) 6862:F8620. doi: 10.1117/12.763449

119. Hirvonen LM, Jiggins S, Sergent N, Zanda G, Suhling K. Photon counting imaging with an electron-bombarded CCD: towards wide-field timecorrelated single photon counting (TCSPC). Nuclear Instrum Methods A. (2015) 787:323-7. doi: 10.1016/j.nima.2015.01.031

120. Hirvonen LM, Barber MJ, Suhling $K$. Photon counting imaging and centroiding with an EBCCD using single molecule localisation software. Nuclear Instrum Methods A. (2016) 820:121-5. doi: 10.1016/j.nima.2016.03.024

121. Barbier R, Baudot J, Chabanat E, et al. Performance study of a MegaPixel single photon position sensitive photodetector EBCMOS. Nuclear Instrum Methods A. (2009) 610:54-6. doi: 10.1016/j.nima.2009.05.054

122. Barbier R, Cajgfinger $T$, Calabria $\mathrm{P}$, et al. A single-photon sensitive ebCMOScamera: the LUSIPHER prototype. Nuclear Instrum Methods A. (2011) 648:266-74. doi: 10.1016/j.nima.2011.04.018

123. Hamamatsu Photonics K. K. Photomultiplier Tubes. 3rd ed. Electron Tube Division (2006).

124. Fisher EMD. Photon counting - fundamentals and applications. In: Britun N, Nikiforov A, editors. Principles and Early Historical Development of Silicon Avalanche and GeigerMode Photodiodes. InTech (2018). doi: 10.5772/intechopen. 72148

125. Holzman I, Ivry Y. Superconducting nanowires for singlephoton detection: progress, challenges, and opportunities.
Adv QTM Technol. (2019) 2:1800058. doi: 10.1002/qute.2018 00058

126. Gol'tsman GN, Okunev O, Chulkova G, Lipatov A, Semenov A, Smirnov K, et al. Picosecond superconducting single-photon optical detector. Appl Phys Lett. (2001) 79:705-7. doi: 10.1063/1.1388868

127. Korzh B, Zhao QY, Allmaras JP, Frasca S, Autry TM, Bersin EA, et al. Demonstration of sub-3 ps temporal resolution with a superconducting nanowire single-photon detector. Nat Photonics. (2020).

128. Irwin KD. An application of electrothermal feedback for high resolution cryogenic particle detection. Appl Phys Lett. (1995) 66:1998-2000. doi: $10.1063 / 1.113674$

129. Eisenhauer F, Raab W. Visible/infrared imaging spectroscopy and energy-resolving detectors. Annu Rev Astro Astrophys. (2015) 53:155-97. doi: 10.1146/annurev-astro-082214-122442

130. Haugen GR, Wallin BW, Lytle FE. Optimization of data-acquistion rates in time-correlated single-photon fluorimetry. Rev Sci Instruments. (1979) 50:64-72. doi: 10.1063/1.1135671

131. Mota M, Christiansen J. A high-resolution time interpolator based on a delay locked loop and an RC delay line. IEEE J Solid Circ. (1999) 34:1360-6. doi: $10.1109 / 4.792603$

132. Lampton M, Raffanti R. A high-speed wide dynamic range time-to-digital converter. Rev Sci Instrum. (1994) 65:3577-584. doi: 10.1063/1.1144540

133. Antonioli S, Cuccato A, Miari L, Labanca I, Rech I, Ghioni M. Ultracompact 32-channel system for time-correlated single-photon counting measurements. Proc SPIE. (2013) 8773:87730D. doi: 10.1117/12.2017408

134. Peronio P, Labanca I, Acconcia G, Ruggeri A, Lavdas AA, Hicks $\mathrm{AA}$, et al. 32-channel time-correlated-single-photon-counting system for high-throughput lifetime imaging. Rev Sci Instrum. (2017) 88:083704. doi: $10.1063 / 1.4986049$

135. Stoppa D, Borghetti F, Richardson JA, Walker R, Henderson RK, Gersbach $\mathrm{M}$, et al. Ultra compact and low-power TDC and TAC architectures for highly-parallel implementation in time-resolved image sensors. In: 2011 International Workshop on ADC Modelling, Testing And Data Converter Analysis and Design and IEEE 2011 ADC Forum (Orvieto) (2011).

136. Krishnan RV, Masuda A, Centonze VFE, Herman BA. Quantitative imaging of protein-protein interactions by multiphoton fluorescence lifetime imaging microscopy using a streak camera. J Biomed Opt. (2003) 8:362-7. doi: $10.1117 / 1.1577574$

137. Krishnan RV, Saitoh H, Terada H, Centonze VE, Herman B. Development of a multiphoton fluorescence lifetime imaging microscopy system using a streak camera. Rev Sci Instrum. (2003) 74:2714-21. doi: 10.1063/1.1569410

138. Biskup C, Zimmer T, Benndorf K. FRET between cardiac $\mathrm{Na}^{+}$channel subunits measured with a confocal microscope and a streak camera. Nat Biotechnol. (2004) 22:220-4. doi: 10.1038/nbt935

139. Biskup C, Hoffmann B, Kelbauskas L, Zimmer T, Klocker N, Becker W, et al. Multidimensional fluorescence lifetime measurements. In: Periasamy A, PTC So, editors. Multiphoton Microscopy in the Biomedical Sciences VIII. Vol. 6860. International Society for Optics and Photonics. Proc SPIE. (2008). p. 260-71. doi: $10.1117 / 12.762834$

140. Komura M, Itoh S. Fluorescence measurement by a streak camera in a single-photon-counting mode. Photosyn Res. (2009) 101:119-33. doi: 10.1007/s11120-009-9463-x

141. Davis LM, Parigger C. Use of streak camera for time-resolved photon counting fluorimetry. Meas Sci Technol. (1992) 3:85-90. doi: $10.1088 / 0957-0233 / 3 / 1 / 012$

142. Godet J, Mély Y. Exploring protein-protein interactions with large differences in protein expression levels using FLIM-FRET. Methods Appl Fluoresc. (2019) 8:014007. doi: 10.1088/2050-6120/ab5dd2

143. Trinh AL, Ber S, Howitt A, Valls PO, Fries MW, Venkitaraman AR, et al. Fast single-cell biochemistry: theory, open source microscopy and applications. Methods Appl Fluoresc. (2019) 7:044001. doi: 10.1088/2050-6120/ab3bd2

144. Lukina MM, Shimolina LE, Kiselev NM, Zagainov VE, Komarov DV, Zagaynova EV, et al. Interrogation of tumor metabolism in tissue samples ex vivo using fluorescence lifetime imaging of $\mathrm{NAD}(\mathrm{P}) \mathrm{H}$. Methods Appl Fluoresc. (2019) 8:014002. doi: 10.1088/2050-6120/ab4ed8

145. Cao R, Wallrabe H, Siller K, Periasamy A. Optimization of FLIM imaging, fitting and analysis for auto-fluorescent $\mathrm{NAD}(\mathrm{P}) \mathrm{H}$ and 
FAD in cells and tissues. Methods Appl Fluoresc. (2020) 8:024001. doi: 10.1088/2050-6120/ab6f25

146. Suhling K, Hirvonen LM, Becker W, Smietana S, Netz H, Milnes J, et al. Wide-field time-correlated single photon counting-based fluorescence lifetime imaging microscopy. Nucl Instrum Meth A. (2019) 942:162365. doi: 10.1016/j.nima.2019.162365

147. McLoskey D, Birch DJS, Sanderson A, Suhling K, Welch E, Hicks PJ. Multiplexed single-photon counting. I. A time-correlated fluorescence lifetime camera. Rev Sci Instrum. (1996) 67:2228-37. doi: 10.1063/1.1147041

148. Rinnenthal JL, Barnchen C, Radbruch H, Andresen V, Mossakowski A, Siffrin V, et al. Parallelized TCSPC for dynamic intravital fluorescence lifetime imaging: quantifying neuronal dysfunction in neuroinflammation. PLoS ONE. (2013) 8:e60100. doi: 10.1371/journal.pone.0060100

149. Levitt JA, Poland SP, Krstajic N, Pfisterer K, Erdogan A, Barber PR, et al. Quantitative real-time imaging of intracellular FRET biosensor dynamics using rapid multi-beam confocal FLIM. Sci Rep. (2020) 10:5146. doi: 10.1038/s41598-020-61478-1

150. Hanley QS, Subramaniam V, Arndt-Jovin DJ, Jovin TM. Fluorescence lifetime imaging: multi-point calibration, minimum resolvable differences, and artifact suppression. Cytometry. (2001) 43:248-60. doi: 10.1002/1097-0320(20010401)43:4<248::AID-CYTO1057>3.0.CO;2-Y

151. Periasamy A, Clegg RM, editors. FLIM Microscopy in Biology and Medicine. Chapman and Hall/CRC (2009). doi: 10.1201/9781420078916

152. Hirvonen LM, Festy F, Suhling K. Wide-field time-correlated single-photon counting (TCSPC) lifetime microscopy with microsecond time resolution. Opt Lett. (2014) 39:5602-5. doi: 10.1364/OL.39.005602

153. Hirvonen LM, Petrášek Z, Beeby A, Suhling K. Sub- $\mu$ s time resolution in wide-field time-correlated single photon counting microscopy obtained from the photon event phosphor decay. N J Phys. (2015) 17:023032. doi: $10.1088 / 1367-2630 / 17 / 2 / 023032$

154. Hirvonen LM, Fisher-Levine M, Suhling K, Nomerotski A. Photon counting phosphorescence lifetime imaging with TimepixCam. Rev Sci Instrum. (2017) 88:013104. doi: 10.1063/1.4973717

155. Sen R, Hirvonen LM, Zhdanov AV, Svihra P, Andersson-Engels S, Nomerotski A, et al. New luminescence lifetime macro-imager based on a Tpx3Cam optical camera. Biomed Opt Express. (2020) 11:77-88. doi: 10.1364/BOE.11.000077

156. Michalet X, Colyer R, Antelman J, Siegmund O, Tremsin A, Vallerga $\mathrm{J}$, et al. Single-quantum dot imaging with a photon counting camera. Curr Pharm Biotechnol. (2009) 10:543-57. doi: 10.2174/13892010978 8922100

157. Hartig R, Prokazov Y, Turbin E, Zuschratter W. Wide-field fluorescence lifetime imaging with multi-anode detectors. Methods Mol Biol. (2014) 1076:457-80. doi: 10.1007/978-1-62703-649-8_20

158. Jagutzki O, Cerezo A, Czasch A, Dorner R, Hattass M, Huang M, et al. Multiple hit read-out of a microchannel plate detector with a three-layer delay-line anode. IEEE Nuclear Sci Conf R. (2001) 2:850-854.

159. Hirvonen LM, Becker W, Milnes J, Conneely T, Smietana S, Le Marois A, et al. Picosecond wide-field time-correlated single photon counting fluorescence microscopy with a delay line anode detector. Appl Phys Lett. (2016) 109:071101. doi: 10.1063/1.4961054

160. Hirvonen LM, Nedbal J, Almutairi N, Phillips TA, Becker W, Conneely $\mathrm{T}$, et al. Lightsheet fluorescence lifetime imaging microscopy with widefield time-correlated single photon counting. J Biophotonics. (2019) 13:e201960099. doi: 10.1002/jbio.201960099

161. Petrasek Z, Eckert HJ, Kemnitz K. Wide-field photon counting fluorescence lifetime imaging microscopy: application to photosynthesizing systems. Photosyn Res. (2009) 102:157-68. doi: 10.1007/s11120-009-9444-0

162. Vitali M, Picazo F, Prokazov Y, Duci A, Turbin E, Götze C, et al. Wide-Field Multi-Parameter FLIM: long-term minimal invasive observation of proteins in living cells. PLoS ONE. (2011) 6:e15820. doi: 10.1371/journal.pone.0015820

163. Poudel C, Mela I, Kaminski CF. High-throughput, multi-parametric, and correlative fluorescence lifetime imaging. Methods Appl Fluoresc. (2020) 8:024005. doi: 10.1088/2050-6120/ab7364

164. Rodimova SA, Meleshina AV, Kalabusheva EP, Dashinimaev EB, Reunov DG, Torgomyan HG, et al. Metabolic activity and intracellular $\mathrm{pH}$ in induced pluripotent stem cells differentiating in dermal and epidermal directions. Methods Appl Fluoresc. (2019) 7:044002. doi: 10.1088/2050-6120/ab3b3d

165. Biskup C, Gensch T. Fluorescence Lifetime Imaging of Ions in Biological Tissues. Boca Raton, FL: CRC Press; 2015. doi: 10.1201/b17018-30

166. Kuimova MK. Mapping viscosity in cells using molecular rotors. Phys Chem Chem Phys. (2012) 14:12671-12686. doi: 10.1039/c2cp41674c

167. Vysniauskas A, Kuimova MK. A twisted tale: measuring viscosity and temperature of microenvironments using molecular rotors. Int Rev Phys Chem. (2018) 37:259-85. doi: 10.1080/0144235X.2018.1510461

168. Okabe K, Inada N, Gota C, Harada Y, Funatsu T, Uchiyama S. Intracellular temperature mapping with a fluorescent polymeric thermometer and fluorescence lifetime imaging microscopy. Nat Commun. (2012) 3:705. doi: 10.1038/ncomms1714

169. Jenkins J, Borisov SM, Papkovsky DB, Dmitriev RI. Sulforhodamine nanothermometer for multiparametric fluorescence lifetime imaging microscopy. Anal Chem. (2016) 88:10566-72. doi: 10.1021/acs.analchem.6b02675

170. Wang Xd, Wolfbeis OS. Optical methods for sensing and imaging oxygen: materials, spectroscopies and applications. Chem Soc Rev. (2014) 43:3666-761. doi: 10.1039/C4CS00039K

171. Papkovsky DB, Dmitriev RI. Imaging of oxygen and hypoxia in cell and tissue samples. Cell Mol Life Sci. (2018) 75:2963-80. doi: $10.1007 / \mathrm{s} 00018-018-2840-\mathrm{x}$

172. Becker W. Fluorescence lifetime imaging-techniques and applications. J Microsc. (2012) 247:119-36. doi: 10.1111/j.1365-2818.2012.03 618.x

173. Berezin MY, Achilefu S. Fluorescence lifetime measurements and biological imaging. Chem Rev. (2010) 110:2641-84. doi: 10.1021/cr90 $0343 z$

174. Borst JW, Visser AJWG. Fluorescence lifetime imaging microscopy in life sciences. Meas Sci Technol. (2010) 21:102002. doi: 10.1088/0957-0233/21/10/102002

175. Chen LC, Lloyd WR, Chang CW, Sud D, Mycek MA. Chapter 20 Fluorescence Lifetime Imaging Microscopy for Quantitative Biological Imaging. In: Sluder G, Wolf DE, editors. Digital Microscopy. Vol. 114 of Methods in Cell Biology. Oxford: Academic Press (2013). p. 457-88. doi: 10.1016/B978-0-12-407761-4.00020-8

176. Baggaley E, Weinstein JA, Williams JAG. Lighting the way to see inside the live cell with luminescent transition metal complexes. Coordin Chem Rev. (2012) 256:1762-85. doi: 10.1016/j.ccr.2012. 03.018

177. Marcu L, French PMW, Elson DS, editors. Fluorescence Lifetime Spectroscopy and Imaging. Boca Raton, FL: CRC Press (2015). doi: 10.1201/b1 7018

178. Suhling K, Hirvonen LM, Levitt JA, et al. Fluorescence lifetime imaging (FLIM): Basic concepts and some recent developments. Med Photonics. (2015) 27:3-40. doi: 10.1016/j.medpho.2014. 12.001

179. Kapusta P, Wahl M, Erdmann R, editors. Advanced Photon Counting: Applications, Methods, Instrumentation. Cham: Springer International Publishing (2015). doi: 10.1007/978-3-319-15636-1

180. Wang S, Shen B, Ren S, Zhao Y, Zhang S, Qu J, et al. Implementation and application of FRET-FLIM technology. J Innov Opt Heal Sci. (2019) 12:1930010. doi: 10.1142/S1793545819300106

181. König K, editor. Multiphoton Microscopy and Fluorescence Lifetime Imaging. Berlin; Boston, MA: De Gruyter (2018). doi: 10.1515/9783110429985.

182. Becker W, Bergmann A, Smietana S. Fast-acquisition TCSPC FLIM with sub25-ps IRF width. In: Periasamy A, PTC So, König K, editors. Multiphoton Microscopy in the Biomedical Sciences XIX. Vol. 10882. Proc SPIE. (2019). p. 1-7. doi: 10.1117/12.2510272

183. Becker W, Bergmann A, Ibarrola RS, Müller PF, Braun L. Metabolic imaging by simultaneous FLIM of NAD(P)H and FAD. In: Periasamy A, PTC So, König K, editors. Multiphoton Microscopy in the Biomedical Sciences XIX. Vol. 10882. Proc SPIE. (2019). p. 34-41. doi: 10.1117/12.2510132

184. Sanchez WY, Pastore M, Haridass IN, König K, Becker W, Roberts MS. "Advanced Time-Correlated Single Photon Counting Applications," in Fluorescence Lifetime Imaging of the Skin, eds Becker W. Vol. 
111. Springer Series in Chemical Physics (Cham: Springer) (2015). doi: 10.1007/978-3-319-14929-5_15

185. Levitt JA, Kuimova MK, Yahioglu G, Chung PH, Suhling K, Phillips D. Membrane-bound molecular rotors measure viscosity in live cells via fluorescence lifetime imaging. J Phys Chem C. (2009) 113:11634-42. doi: $10.1021 /$ jp 9013493

186. Steinmark IE, James AL, Chung PH, Morton PE, Parsons M, Dreiss $\mathrm{CA}$, et al. Targeted fluorescence lifetime probes reveal responsive organelle viscosity and membrane fluidity. PLoS ONE. (2019) 14:e0211165. doi: 10.1371/journal.pone. 0211165

187. Poland SP, Chan GK, Levitt JA, Krstajić N, Erdogan AT, Henderson RK, et al. Multifocal multiphoton volumetric imaging approach for high-speed timeresolved Förster resonance energy transfer imaging in vivo. Opt Lett. (2018) 43:6057-60. doi: 10.1364/OL.43.006057
188. Becker W, Hirvonen LM, Milnes JS, Conneely T, Jagutzki O, Netz H, et al. A wide-field TCSPC FLIM system based on an MCP PMT with a delay-line anode. Rev Sci Instrum. (2016) 87:093710. doi: 10.1063/1.4962864

Conflict of Interest: The authors declare that the research was conducted in the absence of any commercial or financial relationships that could be construed as a potential conflict of interest.

Copyright $\odot 2020$ Hirvonen and Suhling. This is an open-access article distributed under the terms of the Creative Commons Attribution License (CC BY). The use, distribution or reproduction in other forums is permitted, provided the original author(s) and the copyright owner(s) are credited and that the original publication in this journal is cited, in accordance with accepted academic practice. No use, distribution or reproduction is permitted which does not comply with these terms. 